\title{
Drebrin-like (Dbnl) Controls Neuronal Migration via Regulating N-Cadherin Expression in the Developing Cerebral Cortex
}

\author{
Seika Inoue, ${ }^{1}$ Kanehiro Hayashi, ${ }^{1}$ Kyota Fujita, ${ }^{2}$ Kazuhiko Tagawa, ${ }^{2}{ }^{-}$Hitoshi Okazawa, ${ }^{2}$-Ken-ichiro Kubo, ${ }^{1}$ \\ and $\odot$ Kazunori Nakajima ${ }^{1}$ \\ ${ }^{1}$ Department of Anatomy, Keio University School of Medicine, Tokyo 160-8582, Japan, and 2Department of Neuropathology, Medical Research Institute, \\ Center for Brain Integration Research, Tokyo Medical and Dental University, Tokyo 113-8510, Japan
}

The actin cytoskeleton is crucial for neuronal migration in the mammalian developing cerebral cortex. The adaptor protein Drebrin-like (Dbnl) plays important roles in reorganization of the actin cytoskeleton, dendrite formation, and endocytosis by interacting with F-actin, cobl, and dynamin. Although Dbnl is known to be expressed in the brain, the functions of this molecule during brain development are largely unknown. In this study, to examine the roles of Dbnl in the developing cerebral cortex, we conducted experiments using mice of both sexes with knockdown of Dbnl, effected by in utero electroporation, in the migrating neurons of the embryonic cortex. Time-lapse imaging of the Dbnl-knockdown neurons revealed that the presence of Dbnl is a prerequisite for appropriate formation of processes in the multipolar neurons in the multipolar cell accumulation zone or the deep part of the subventricular zone, and for neuronal polarization and entry into the cortical plate. We found that Dbnl knockdown decreased the amount of N-cadherin protein expressed on the plasma membrane of the cortical neurons. The defect in neuronal migration caused by Dbnl knockdown was rescued by moderate overexpression of N-cadherin and $\alpha \mathrm{N}$-catenin or by transfection of the phospho-mimic form (Y337E, Y347E), but not the phospho-resistant form (Y337F, Y347F), of Dbnl. These results suggest that Dbnl controls neuronal migration, neuronal multipolar morphology, and cell polarity in the developing cerebral cortex via regulating $\mathrm{N}$-cadherin expression.

Key words: cerebral cortex; development; neuronal migration; phosphorylation

Significance Statement

Disruption of neuronal migration can cause neuronal disorders, such as lissencephaly and subcortical band heterotopia. During cerebral cortical development, the actin cytoskeleton plays a key role in neuronal migration; however, the mechanisms of regulation of neuronal migration by the actin cytoskeleton still remain unclear. Herein, we report that the novel protein Dbnl, an actin-binding protein, controls multiple events during neuronal migration in the developing mouse cerebral cortex. We also showed that this regulation is mediated by phosphorylation of Dbnl at tyrosine residues 337 and 347 and $\alpha \mathrm{N}$-catenin/N-cadherin, suggesting that the Dbnl- $\alpha \mathrm{N}$-catenin/N-cadherin pathway is important for neuronal migration in the developing cortex.

\section{Introduction}

In the developing mammalian cerebral cortex, projection neurons are generated in the ventricular zone (VZ) or subventricular

Received June 29, 2018; revised Nov. 3, 2018; accepted Nov. 15, 2018.

Author contributions: S.I. and K.H. wrote the first draft of the paper; S.I., K.H., K.-i.K., and K.N. edited the paper; S.I., K.H., K.F., K.T., H.O., K.-i.K., and K.N. designed research; S.I., K.H., K.F., K.T., and H.O. performed research; S.I. contributed unpublished reagents/analytic tools; S.I., K.H., K.F., K.T., H.O., K.-i.K., and K.N. analyzed data; S.I., K.H., K.-i.K., and K.N. wrote the paper.

This work was supported by Ishidsu Shun Memorial Scholarship, Japan; Ministry of Education, Culture, Sports, Science and Technology/Japan Society for the Promotion of Science Grants-in-Aid for Scientific Research (KAKENHI) JP16H06482, JP15H02355, JP15K06745, JP18K06842, and JP18K07855; Naito Foundation; Takeda Science Foundation; Novartis Foundation (Japan) for the Promotion of Science; Kawano Masanori Memorial Public Interest Incorporated Foundation for Promotion of Pediatrics; Keio Gijuku Academic Development Funds; and Keio Gijuku Fukuzawa Memorial Fund for the Advancement of Education and Research. We thank Prof. Huganir and Prof. zone (SVZ), and many of these then sojourn to the multipolar cell accumulation zone (MAZ), which overlaps with the deeper part of the SVZ (Tabata and Nakajima, 2003; Noctor et al., 2004; Tabata et al., 2009). In this zone, the neurons assume multipolar morphologies and exhibit dynamic elongation and retraction of their multiple thin processes (multipolar migration) (Tabata and

Sakagami for providing the mouse $\mathrm{N}$-cadherin CDNA and the guinea pig anti-N-cadherin antibody, respectively; and Collaborative Research Resources, Keio University School of Medicine, for technical support and supply of reagents. The authors declare no competing financial interests.

Correspondence should be addressed to Kazunori Nakajima at kazunori@keio.jp.

https://doi.org/10.1523/JNEUROSCI.1634-18.2018

Copyright $\odot 2019$ the authors $\quad 0270-6474 / 19 / 390678-14 \$ 15.00 / 0$ 
Table 1. Details of the plasmid DNA concentrations

\begin{tabular}{|c|c|c|c|c|c|c|c|c|c|c|c|}
\hline & Figure & Panel & Vector & $\begin{array}{l}\text { Concentration } \\
(\mathrm{mg} / \mathrm{ml})\end{array}$ & Vector & $\begin{array}{l}\text { Concentration } \\
(\mathrm{mg} / \mathrm{ml})\end{array}$ & Vector & $\begin{array}{l}\text { Concentration } \\
(\mathrm{mg} / \mathrm{ml})\end{array}$ & Vector & $\begin{array}{l}\text { Concentration } \\
(\mathrm{mg} / \mathrm{ml})\end{array}$ & $\begin{array}{l}\text { Total } \\
\text { concentration } \\
(\mathrm{mg} / \mathrm{ml})\end{array}$ \\
\hline \multirow[t]{2}{*}{ In vitro } & 2 & $C$ & pCAGGS-EGFP & 0.5 & pSilencer 3.0 & 8 & - & - & - & - & 8.5 \\
\hline & & & pCAGGS-EGFP & 0.5 & pSilencer-Dbnl & 8 & - & - & - & - & 8.5 \\
\hline \multirow[t]{2}{*}{ In vivo } & & $E, G$ & pCAGGS-EGFP & 2 & pSilencer 3.0 & 2 & - & - & - & - & 4 \\
\hline & & & pCAGGS-EGFP & 2 & pSilencer-Dbnl & 2 & - & - & - & - & 4 \\
\hline \multirow[t]{5}{*}{ In vivo } & & $L$ & pCAGGS-EGFP & 2 & pSilencer 3.0 & 2 & pCAGGS1 & 0.5 & - & - & 4.5 \\
\hline & & & pCAGGS-EGFP & 2 & pSilencer-Dbnl & 2 & pCAGGS1 & 0.5 & - & - & 4.5 \\
\hline & & & pCAGGS-EGFP & 2 & pSilencer-Dbnl & 2 & pCAG-Dbnl resistance & 0.5 & - & - & 4.5 \\
\hline & & & pCAGGS-EGFP & 2 & pSilencer-Dbnl & 2 & pCAG-Dbnl 2F & 0.5 & - & - & 4.5 \\
\hline & & & pCAGGS-EGFP & 2 & pSilencer-Dbnl & 2 & pCAG-Dbnl 2E & 0.5 & - & - & 4.5 \\
\hline \multirow[t]{2}{*}{ In vivo } & 3 & $A, C$ & pCAGGS-EGFP & 2 & pSilencer 3.0 & 2 & 一 & - & - & - & 4 \\
\hline & & & pCAGGS-EGFP & 2 & pSilencer-Dbnl & 2 & - & - & - & - & 4 \\
\hline \multirow[t]{2}{*}{ In vivo } & 4 & $A, F, J, N$ & pCALNL-EGFP & 2 & pCAGGS-M-cre & 0.002 & pSilencer 3.0 & 2 & - & - & 4.002 \\
\hline & & $A^{\prime}, F^{\prime}, J^{\prime}, N^{\prime}$ & pCALNL-EGFP & 2 & pCAGGS-M-cre & 0.002 & pSilencer-Dbnl & 2 & - & - & 4.002 \\
\hline \multirow[t]{2}{*}{ In vivo } & 5 & $A, C$ & pCAGGS-EGFP & 2 & pSilencer 3.0 & 2 & - & - & - & - & 4 \\
\hline & & $B, D$ & pCAGGS-EGFP & 2 & pSilencer-Dbnl & 2 & - & - & - & - & 4 \\
\hline \multirow[t]{2}{*}{ In vitro } & 6 & $A$ & pCAGGS-EGFP & 0.5 & pSilencer 3.0 & 8 & - & - & - & - & 8.5 \\
\hline & & & pCAGGS-EGFP & 0.5 & pSilencer-Dbnl & 8 & - & - & - & - & 8.5 \\
\hline \multirow[t]{5}{*}{ In vivo } & & $C$ & pCAGGS-EGFP & 2 & pSilencer 3.0 & 2 & pCAGGS1 & 0.5 & - & - & 4.5 \\
\hline & & & pCAGGS-EGFP & 2 & pSilencer-Dbnl & 2 & pCAGGS1 & 0.5 & - & - & 4.5 \\
\hline & & & pCAGGS-EGFP & 2 & pSilencer-Dbnl & 2 & pCAG-N-caherin & 0.5 & - & - & 4.5 \\
\hline & & & pCAGGS-EGFP & 2 & pSilencer-Dbnl & 2 & $\mathrm{pCAG}-\alpha \mathrm{N}$-catenin & 0.5 & - & - & 4.5 \\
\hline & & & pCAGGS-EGFP & 2 & pSilencer-Dbnl & 2 & pCAG-N-caherin & 0.25 & pCAG- $\alpha \mathrm{N}$-catenin & 0.25 & 4.5 \\
\hline
\end{tabular}
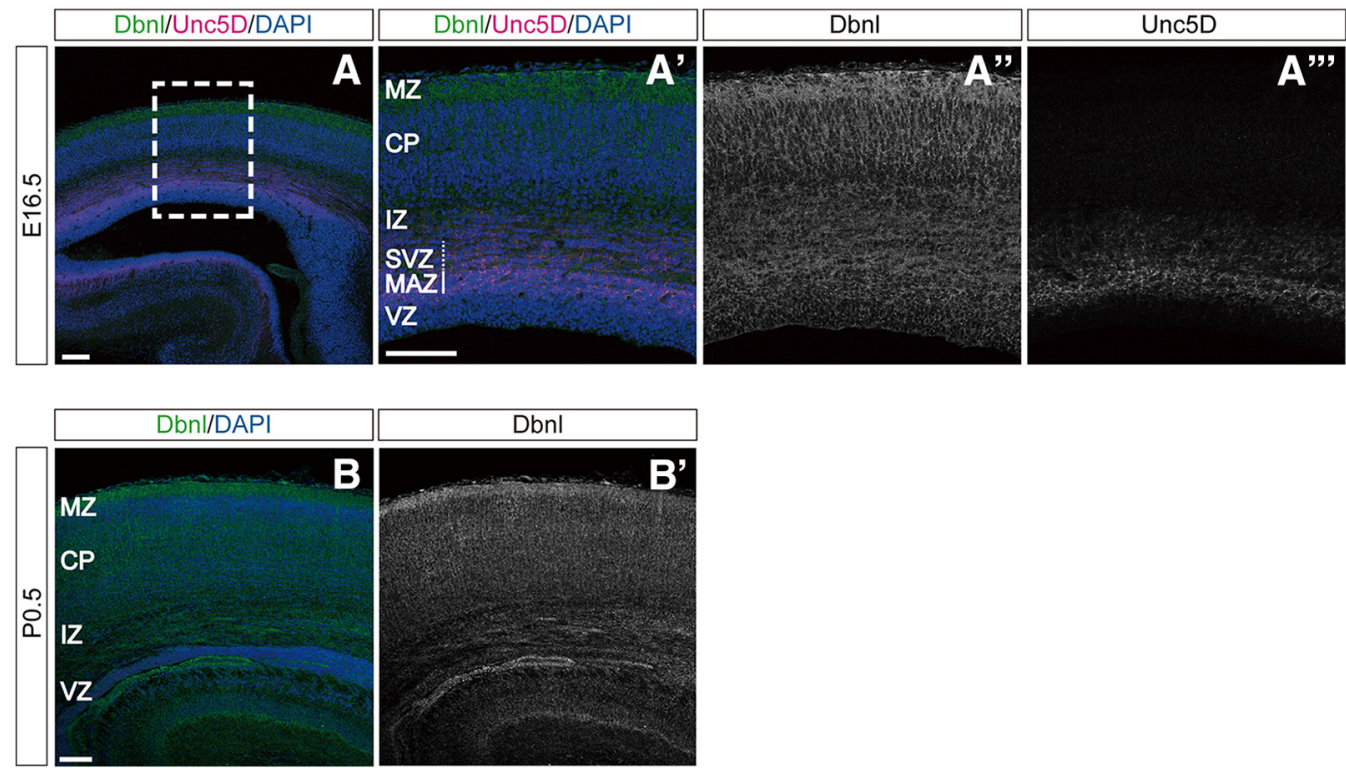

Figure 1. Dbnl is expressed in the developing mouse cerebral cortex. $A, B$, Immunohistochemical staining for Dbnl (green), Unc5D (magenta) as a marker for the MAZ/SVZ ( $\left.A^{\prime \prime \prime}\right)$, and DAPI (blue) using sections from the cerebral cortex at E16.5 $\left(\boldsymbol{A}-\boldsymbol{A}^{\prime \prime \prime}\right)$ and P0.5 $\left(\boldsymbol{B}, \boldsymbol{B}^{\prime}\right)$ of the ICR mouse. Scale bars, $100 \mu \mathrm{m}$.

Nakajima, 2003; Noctor et al., 2004). Subsequently, the multipolar cells transform into bipolar cells and start to migrate along with the radial glial fibers through the intermediate zone (IZ) to the cortical plate (CP) (locomotion mode) (Rakic, 1972; Nadarajah et al., 2001). Finally, when the locomoting neurons reach the primitive cortical zone (PCZ), the outermost region of the $\mathrm{CP}$ beneath the marginal zone (MZ) (Sekine et al., 2011), they quickly move their somata to just beneath the MZ, through shortening of their leading processes, while retaining contact with the MZ at their tip (terminal translocation mode), to complete their migration (Nadarajah et al., 2001; Franco et al., 2011; Sekine et al., 2011, 2012).
Actin-associated proteins play important roles in neuronal migration by regulating the polymerization and depolymerization of actin. The actin-depolymerizing factor homology (ADF-H) domain is crucial for actin dynamics (Vartiainen et al., 2002; Goroncy et al., 2009). This domain is present in three phylogenetically distinct classes of actin-binding proteins: the ADF/ cofilins, the twinfilins, and the Abp1/drebrins (Lappalainen et al., 1998). Drebrin-like, a member of the Abp1/drebrins family (Dbnl; also referred to as mAbp1, HIP-55, or SH3P7) contains the ADF-H domain, a proline-rich region, and the $\mathrm{SH} 3$ domain (Larbolette et al., 1999) (see Fig. 2I). Dbnl plays important roles in reorganization of the actin cytoskeleton, endocytosis, and 


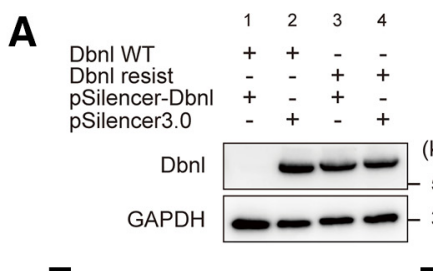

E

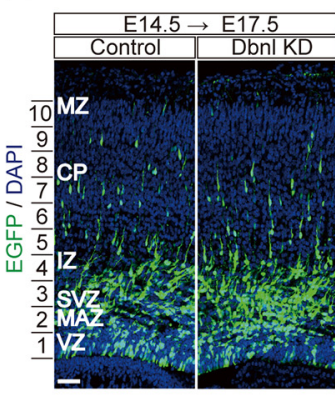

I

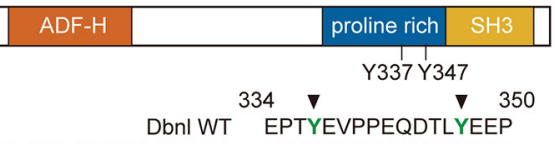

Dbnl 2F (Y337F, Y347F) EPTFEVPPEQDTLFEEP
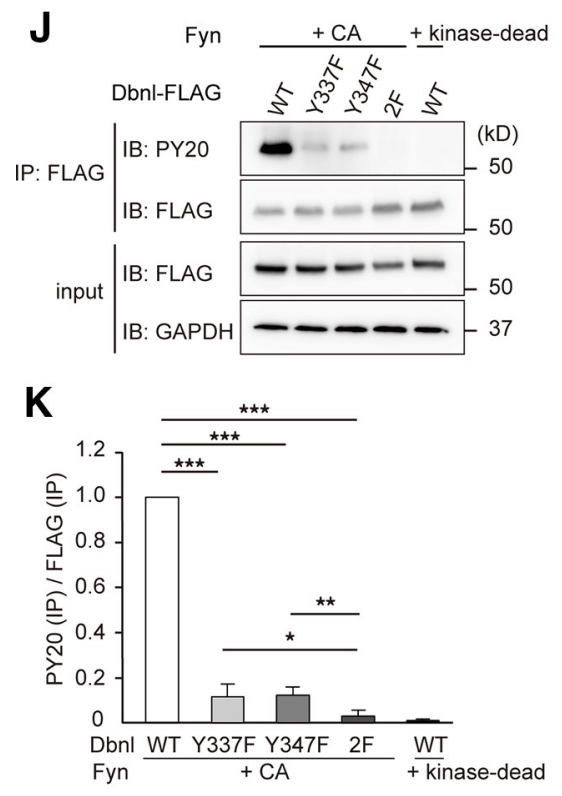

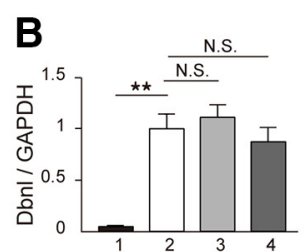

\section{$\mathbf{F}$}

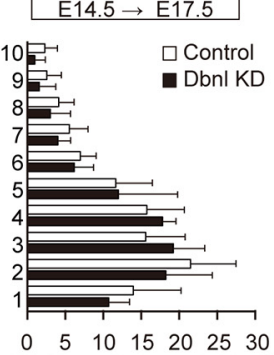

$\begin{array}{ccccccc}0 & 5 & 10 & 15 & 20 & 25 & 30 \\ \text { EGFP+ cells in each bin (\%) }\end{array}$

\section{C}

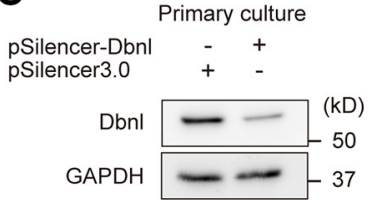

H

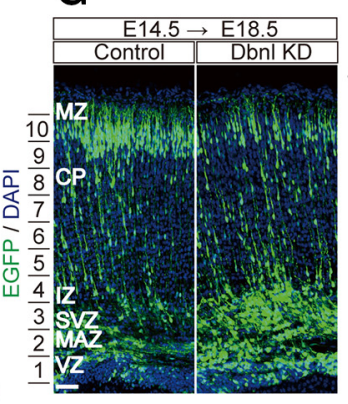

D

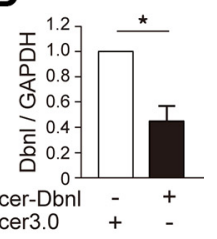

L

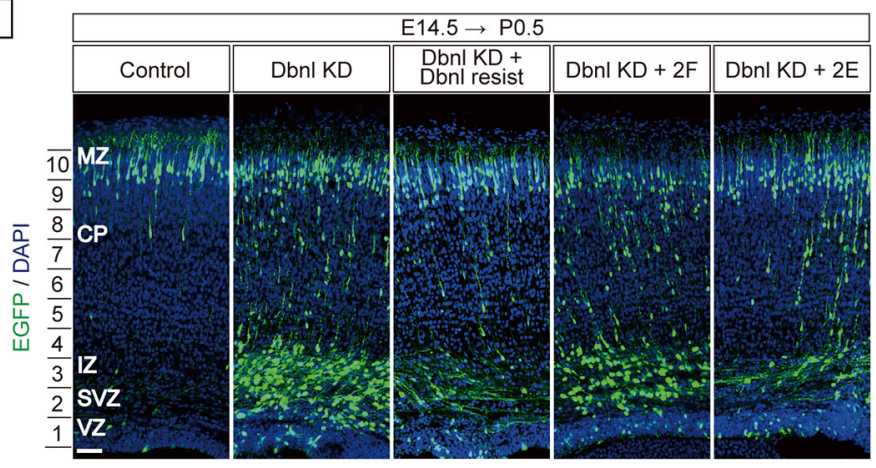

M

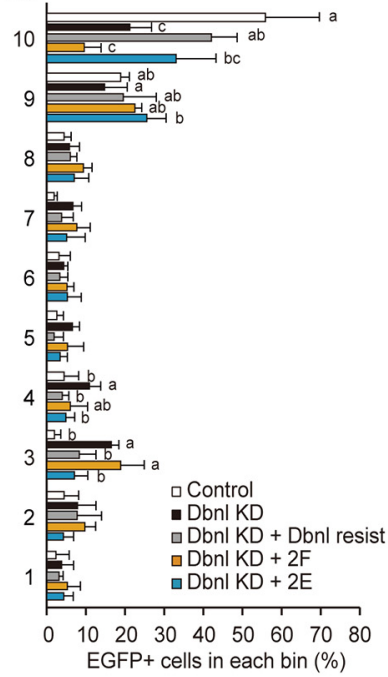

$\mathbf{N}$
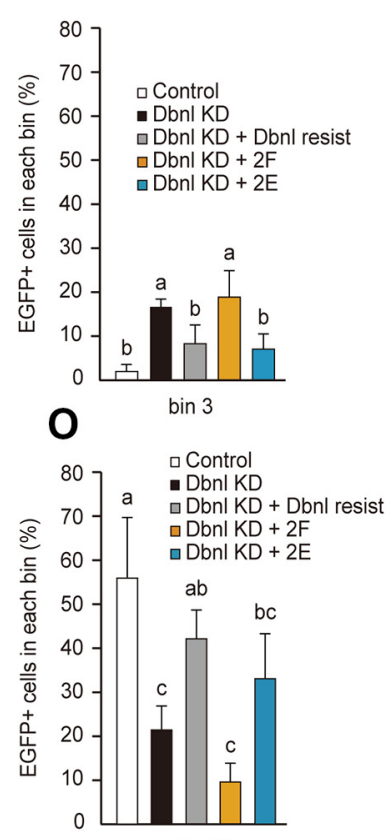

bin 10

Figure 2. Dbnl controls neuronal migration in the MAZ/SVZ and IZ. A, KD of Dbnl by the pSilencer-Dbnl shRNA. The pSilencer-Dbnl shRNA with the pCAGGS-Dbnl-WT or -resist, which was a KD resistant form of Dbnl, was transfected into the HEK293T cells ( $N=3$ experiments). The cell lysates were subjected to Western blotting for Dbnl, with GAPDH measured as the internal control. $\boldsymbol{B}$, A normalized graph represents the Dbnl/GAPDH ratio $(\boldsymbol{A})(N=3$ experiments). $C$, pSilencer-Dbnl shRNA or the empty vector was introduced into the primary culture of cortical neurons. The cell lysates were subjected to Western blotting for Dbnl, with GAPDH measured as the internal control. $D$, A normalized graph represents the Dbnl/GAPDH ratio (C) (N $=3$ experiments). $\boldsymbol{E}$, G, Effects of Dbnl KD on the migration of cortical neurons. In utero electroporation of the mouse embryonic brains at E14.5 with Dbnl-shRNA plus pCAGGS-EGFP, or the pSilencer-control vector plus pCAGGS-EGFP, as control, was performed. There were no obvious differences in position between the control and Dbnl KD neurons at E17.5 (E; Control: $N=10$ brains; (Figure legend continues.) 
dendrite formation by interacting with F-actin, Dynamin, and Cobl (Kessels et al., 2000, 2001; Haag et al., 2012). Furthermore, the phosphorylation of Dbnl by Src kinase at tyrosine residues 337 and 347 in the proline-rich region of this protein is critical for the function (Boateng et al., 2012). While Dbnl is known to be expressed in the brain (Larbolette et al., 1999), the roles of this protein during cortical development still remain unclear.

During cortical development, the Src family kinases (SFKs), which are nonreceptor protein tyrosine kinases, play important roles in many cellular events, such as cell growth, differentiation, adhesion, and migration (Stein et al., 1994; Nam et al., 2005). Although Src, Fyn, and Yes, all members of the SFKs, have been detected in the mammalian developing brain (Cotton and Brugge, 1983; Martinez et al., 1987; Sudol et al., 1988; Cooke and Perlmutter, 1989), Src- and Yes-knock-out mice have been found not to exhibit any serious defects during cortical development (Soriano et al., 1991; Stein et al., 1994). In contrast, deletion of Fyn kinase in the mouse brain has been shown to lead to defective cortical development. For example, Fyn-knock-out mice show abnormal dendritic morphology, misorientation of the cortical pyramidal neurons in layer V (Sasaki et al., 2002), and dislocation of later-generated cortical neurons (Yuasa et al., 2004). These findings suggest that Fyn, among the SFKs, plays the dominant role in the developing cerebral cortex and is essential for the formation of a normal brain architecture. However, the molecular mechanisms underlying the actions of Fyn in the developing neocortex are not yet fully understood.

\footnotetext{
(Figure legend continued.) Dbnl KD: $N=4$ brains), whereas Dbnl KD suppressed migration of the cortical neurons at $4 \mathrm{~d}$ after transfection ( $G$; Control, $N=7$ brains; Dbnl KD, $N=8$ brains). $\boldsymbol{F}, \boldsymbol{H}$, Graphs represent proportion of EGFP-positive cells in each bin. White bar represents Control. Black bar represents Dbnl KD. The entire cortex in the image was divided into 10 equally spaced bins (bin 1, deepest; bin 10, most superficial). Statistically significant differences were observed in bin 3 (Control vs Dbnl KD, ${ }^{* *} p=0.001$ ) and bin 10 (Control vs Dbnl KD, ${ }^{* *} p=$ 0.009) $(\boldsymbol{H}) . \boldsymbol{I}$, Domain structure of Dbnl. Arrowheads on the sequence indicate Y337 and Y347 (green letters), which are the residues phosphorylated by Fyn. Dbnl $2 F$ indicates the Dbnl mutant in which the tyrosine residues Y337 and Y347 were substituted with phenylalanine (magenta letters). J, Y337 and Y347 residues of Dbnl are important for the tyrosine residues phosphorylated by Fyn. Fyn [a constitutively active (CA) or kinase-dead] and Dbnl (WT, Y337F, Y347F, or 2F)-Flag were exogenously coexpressed in the HEK293T cells, and samples immunoprecipitated with the anti-FLAG antibody were subjected to Western blotting with anti-PY20 and FLAG antibodies, while the input was blotted with anti-FLAG and GAPDH antibodies. $\boldsymbol{K}$, A normalized graph represents the PY20/FLAG ratio $(J)(N=6$ experiments). L, Mouse embryonic brains at E14.5 were electroporated with Dbnl KD plus either pCAGGS-1 ( $N=5$ brains), pCAGGS-Dbnl-resist ( $N=5$ brains), pCAGGS-Dbnl $2 F(N=4$ brains), or pCAGGS-Dbnl 2 E $(N=$ 7 brains), together with pCAGGS-EGFP. The pSilencer-control vector plus pCAGGS-1 and pCAGGS-EGFP were transfected as control ( $N=5$ brains). The brains were fixed at P0.5 and sectioned. Each section was stained with DAPI. $\boldsymbol{M}$, Graph represents the proportion of EGFPpositive cells in each bin. White bar represents Control. Black bar represents Dbnl KD. Gray bar represents Dbnl KD + resist [Dbnl rescue]. Orange bar represents Dbnl KD $+2 \mathrm{~F}$. Blue bar represents $D b n l K D+2 E$. The entire cortex in each image was divided into 10 equally spaced bins (bin 1, deepest; bin 10, most superficial). Statistically significant differences were observed in bin 3 (Control vs Dbnl KD, ${ }^{* * *} p<0.001$; Control vs Dbnl 2F, ${ }^{*} p<0.001$; Dbnl rescue vs Dbnl $\mathrm{KD},{ }^{*} p=0.028$; Dbnl rescue vs Dbnl 2F, ${ }^{* *} p=0.007$; Dbnl 2Evs Dbnl KD, ${ }^{* *} p=0.005$; Dbnl $2 \mathrm{E}$ vs Dbnl 2F, ${ }^{*} p=0.01$ ), bin 4 (Control vs Dbnl KD, ${ }^{*} p=0.037$; Dbnl KD vs Dbnl rescue, ${ }^{*} p=$ 0.022 ; Dbnl KD vs Dbnl $2 \mathrm{E},{ }^{*} p=0.034$ ), bin 9 (Dbnl KD vs Dbnl $2 \mathrm{E},{ }^{*} p=0.038$ ), and bin 10 (Control vs Dbnl KD, ${ }^{* * *} p<0.001$; Control vs Dbnl 2F, ${ }^{*} p<0.001$; Control vs Dbnl 2E, ${ }^{* *} p=$ 0.007 ; Dbnl rescue vs Dbnl 2F, $\left.{ }^{* *} p=0.001\right) . \mathbf{N}, \mathbf{O}$, Graphs represent the proportion of EGFPpositive cells in bin $3(\boldsymbol{N})$ and bin $10(\mathbf{O})$. $\boldsymbol{M}$, Different letters on the bars within the same bin indicate a statistically significant difference between the pair, whereas the same letter within the same bin or the absence of any letters within the bins indicates the absence of any statistically significant difference. Error bar indicates mean \pm SEM. Student's $t$ test, Mann-Whitney's $U$ test, or one-way ANOVA with Tukey-Kramer test: ${ }^{*} p<0.05 ;{ }^{* *} p<0.01$; ${ }^{* * *} p<0.001$. Scale bars, $50 \mu \mathrm{m}$.
}

In this study, we demonstrated that Dbnl is required for neuronal radial migration, neuronal process formation during multipolar migration, and polarization of migrating neurons in the MAZ/SVZ and IZ. The function of Dbnl in regulating neuronal migration is dependent on phosphorylation of Dbnl at tyrosine residues 337 and 347. Dbnl upregulates the amount of $\mathrm{N}$-cadherin protein expressed on the neuronal plasma membrane, which is essential for neuronal migration in the developing cerebral cortex.

\section{Materials and Methods}

Animals. Pregnant ICR mice were purchased from Japan SLC. All animal experiments were implemented under the oversight of the Keio University Institutional Animal Care and Use Committee, in compliance with the Institutional Guidelines on Animal Experimentation at Keio University. The day of vaginal plug detection was identified as embryonic day 0 (E0). "E14.0" refers to the morning of embryonic day 14, and "E14.5" refers to the afternoon of embryonic day 14 . Mice of both sexes were used in this study.

Construction of plasmids. The pCAGGS-EGFP plasmid was constructed by inserting the EGFP cDNA into the pCAGGS vector (Niwa et al., 1991).

Gene knockdown (KD) was accomplished by RNA interference using the $\mathrm{pSilencer}$ 3.0-H1 vector (Ambion) containing the H1 RNA promoter for the expression of a short hairpin RNA (shRNA). The shRNA target sense sequences for $\mathrm{Dbnl}$ were as follows: $5^{\prime}$-gatccGCAGAAGCAAC TCACTCAAttcaagagaTTGAGTGAGTTGCTTCTGCttttttggaaa-3', and 5' -gatccGCAGAAGCAACTCACTCAAttcaagagaTTGAGTGAGTTGCT TCTGCttttttggaaa-3'.

For amplifying the $D b n l$ cDNA by PCR, we used the following primers and template: forward primer, containing an EcoRI site: $5^{\prime}$-gcacagaattc gccaccatggcggtgaacctg- $3^{\prime}$; reverse primer, containing a NotI site: $5^{\prime}$ ttgcggccgc tcactctatgagctccacgtagttg- $3^{\prime}$; and template: a FANTOM RIKEN full-length cDNA clone (AK146920). For effecting expression of Dbnl, the PCR product was subcloned into the pCAGGS vector.

The vector expressing a resistant form of Dbnl cDNA against the Dbnl-KD vector (pCAGGS-Dbnl resist) was generated with 3 point mutations by PCR. The primers used were as follows: sense primer: ccttcctgcaggagcaattccctcaaccagaaac; antisense primer: gtttctggttgagggaattgct cctgcaggaagg (bold letters denote the mutated nucleotides).

The phospho-resistant mutant form of Dbnl at Y337 and/or Y347 (Dbnl Y337F, Y347F, or 2F) was generated by PCR using the cDNA encoding Dbnl-resist as the template. The primers used were as follows: sense primer for Y337F, caggaggccttcgtgcgcgtagtcaccgagaaatc; antisense primer for $\mathrm{Y} 337 \mathrm{~F}$, gatttctcggtgactacgcgcacgaaggcctcctg; sense primer for Y347F, cgtgagcagcgcttccaggaacagcac; and antisense primer for Y347F, gtgctgttcctggaagcgctgctcacg (bold letters denote the mutated nucleotides).

The phospho-mimic form of Dbnl with substitution of the Y337 and Y347 residues with glutamic acid (Dbnl 2E) was commercially synthesized by Integrated DNA Technologies (bold letters denote the mutated nucleotides) (gaattcgccaccatggcggtg ... gaacctacagaagaagtacccccagagca ggacaccctcgaagaggaacca ... ctcatagagtgagcggccgc). The cDNA was subcloned into the pCAGGS vector (pCAGGS-Dbnl 2E). The FLAG-tagged Dbnl, Dbnl Y337F, Dbnl Y347F, or Dbnl 2F was subcloned into the pCAGGS vector (pCAGGS-Dbnl-FLAG, pCAGGS-Dbnl Y337FFLAG, pCAGGS-Dbnl Y347F-FLAG, and pCAGGS-Dbnl 2F-FLAG, respectively)

The pCALNL-EGFP plasmid was generated by subcloning EGFP cDNA into the pCALNL vector purchased from Addgene (plasmid 13769) (Matsuda and Cepko, 2007).

The pCAGGS-M-cre was described previously (Kitazawa et al., 2014).

Construction of both pCAGGS-Fyn constitutive active (CA) (the Y531 residue was substituted with phenylalanine) and the pCAGGS-Fyn kinase-dead (the K299 residue was substituted with methionine) has been described previously (Honda and Nakajima, 2006).

The cDNA for $\alpha N$-catenin (Ctnna2) was amplified by PCR using a cDNA library of mouse postnatal day $(\mathrm{P}) 0$ brain as the template, which 
was synthesized using the Superscript III FirstStrand Synthesis System (Thermo Fisher Scientific). The following primers were used for the PCRs: 5'-CGCTCGAGGCCACCATGA CTTCGGCAACTTCACC-3' (5'-XhoI-Ctnna2$\left.3^{\prime}\right)$ and $5^{\prime}$-GCGGCGGCCGCCTAGAAGGAA TCCATTGCCTTG-3' (5'-NotI-Ctnna2-3'). The cDNA was subcloned into the pCAGGS vector.

The WT full-length mouse $\mathrm{N}$-cadherin cDNA [kindly provided by Prof. Huganir (Nuriya and Huganir, 2006)] with an HA tag at its $\mathrm{C}$ terminus was subcloned into the pCAGGS1 vector (pCAGGS-N-cadherin-HA).

These cDNAs were verified by DNA sequencing, and the protein products were confirmed by Western blot analysis.

In utero electroporation. Pregnant ICR mice were deeply anesthetized with pentobarbital sodium (Kyoritsu Seiyaku) administered by intraperitoneal injection, and their intrauterine embryos were surgically manipulated as described previously (Nakajima et al., 1997; Tabata and Nakajima, 2001, 2008). The conditions for the electronic pulses were as follows: $34 \mathrm{~V}, 50 \mathrm{~ms}$, four times using an electroporator (CUY21; Nepagene). For the sparse labeling, we cotransfected pCALNL-EGFP and pCAGGS-M-cre (Kitazawa et al., 2014). Details of the plasmid DNA concentrations for electroporation are described in Table 1.

In vitro electroporation. ICR mouse cortices at E16 were dissected in HBSS, and the cortical cells were dissociated with Papain (Nakalai Tesque). In vitro electroporation was performed using the dissociated cells at a density of $1 \times 10^{7}$ cells per milliliter in Opti-MEM (Opti-MEM I Reduced Serum Medium, Thermo Fisher Scientific) with an electroporator (NEPA21; Nepagene), under the following conditions: porting pulse; $275 \mathrm{~V}, 0.5 \mathrm{~ms}$ length, $50 \mathrm{~ms}$ interval, $2 \times, 10$ decay rate; transfer pulse; $20 \mathrm{~V}, 50 \mathrm{~ms}$ length, $50 \mathrm{~ms}$ interval, $5 \times$, 40 decay rate. After the electroporation, the cells were seeded on to $60 \mathrm{~mm}$ dishes in Neurobasal medium containing $10 \% \mathrm{FBS}$, and incubated at $37^{\circ} \mathrm{C}$ in $5 \% \mathrm{CO}_{2}$ for $2 \mathrm{~h}$. Then, the medium was replaced with Neurobasal medium containing $3 \% \quad \mathrm{~B} 27$ and $0.4 \mathrm{~mm}$ L-glutamine, and the dishes were incubated at $37^{\circ} \mathrm{C}$ in a humidified $5 \% \mathrm{CO}_{2}$ atmosphere for $2 \mathrm{~d}$.

Antibodies. The anti-Dbnl antibody used for the study (catalog \#13015-1-AP, rabbit) was purchased from Proteintech, the anti-Unc5D antibody (catalog \#AF1429, goat) from R\&D Systems, the anti-GAPDH antibody (catalog \#5174, rabbit) from Cell Signaling Technology, and the antibody directed against GM130 (catalog \#610822, mouse), and anti-phosphotyrosine antibody conjugated with HRP (PY20, catalog \#610012, mouse) from BD Biosciences; the antibodies used against FLAG (catalog \#F3165, mouse; catalog \#F7425, rabbit) were purchased from Sigma-Aldrich. The anti-N-cadherin antibody (guinea pig) was kindly provided by Prof. Sakagami (Hara et al., 2016).

Preparation of frozen sections and immunohistochemistry. The embryos and neonates were placed on ice for anesthesia and perfused with 4\% PFA in a $0.1 \mathrm{M}$ sodium phosphate buffer, $\mathrm{pH}$ 7.4. Subsequently, the embryonic brains were removed and postfixed in the same fixative for $12 \mathrm{~h}$ at $4^{\circ} \mathrm{C}$. The brains were frozen in OTC compound (Sakura Finetek) and cut into
B

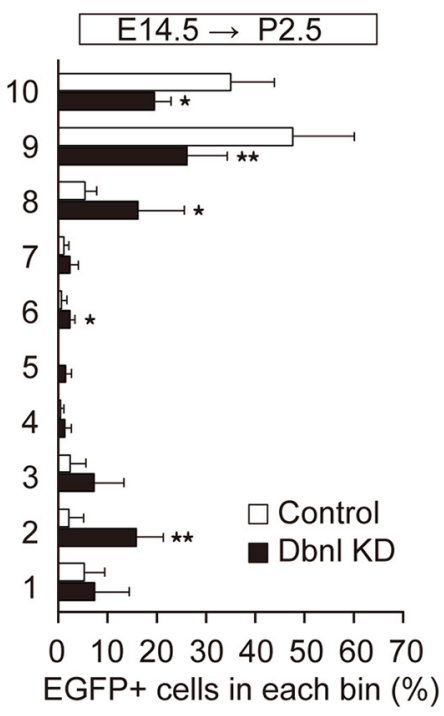

D

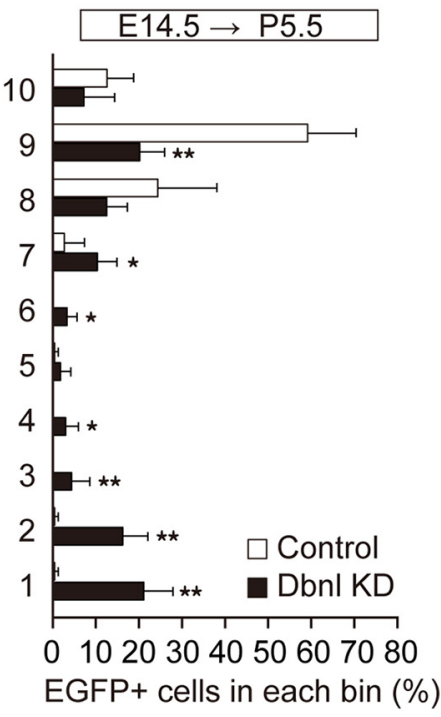

Figure 3. Dbnl controls the neuronal cell positioning after birth. $A, C$, Mouse embryonic brains at E14.5 were electroporated with Dbnl-KD and pCAGGS-EGFP, or pSilencer-control vector and pCAGGS-EGFP as a control, then fixed at P2.5 ( $\boldsymbol{A}$; control: $N=7$ brains; Dbnl KD: $N=5$ brains), or P5.5 (C; control: $N=6$ brains; Dbnl KD: $N=4$ brains). $B, D$, Graphs represent proportion of EGFP-positive cells in each bin. White bar represents Control. Black bar represents Dbnl KD. The entire cortex in the image was divided into 10 equally spaced bins (bin 1, deepest; bin 10, most superficial). Statistically significant differences were observed in bin 2 (Control vs Dbnl KD, ${ }^{* *} p=0.003$ ), bin 6 (Control vs Dbnl KD, ${ }^{*} p=0.048$ ), bin 8 (Control vs Dbnl KD, ${ }^{*} p=0.048$ ), bin 9 2 (Control vs Dbnl KD, ${ }^{* *} p=0.004$ ), bin 3 (Control vs Dbnl KD, ${ }^{* *} p=0.004$ ), bin 4 (Control vs Dbnl KD, ${ }^{*} p=0.03$ ), bin 6 (Control vs Dbnl KD, ${ }^{*} p=0.03$ ), bin 7 (Control vs Dbnl KD, ${ }^{*} p=0.03$ ), and bin 9 (Control vs Dbnl KD, $\left.{ }^{* *} p=0.004\right)$ (D). Error bar indicates mean \pm SEM. Student's $t$ test or Mann-Whitney's $U$ test: ${ }^{*} p<0.05 ;{ }^{* *} p<0.01$. Scale bars, $50 \mu \mathrm{m}$.

$20-\mu$ m-thick sections with a cryostat (CM3050 S, Leica Microsystems). The sections were washed 3 times with PBS containing $0.01 \%$ Triton X-100 (PBS-Tx), then blocked with 10\% normal donkey serum in PBS-Tx at room temperature for $1 \mathrm{~h}$. The sections were incubated with the primary antibodies overnight at $4^{\circ} \mathrm{C}$, then washed 3 times with PBS-Tx and incubated with anti-IgG conjugated with AlexaFlour-594 (Thermo Fisher Scientific) at room temperature for $1 \mathrm{~h}$. Then, DAPI (1:500) was used to stain the nuclei. Images of the immunostained sections were taken under a confocal microscope (FV1000, Olympus; or SP8, Leica Microsystems). The primary antibodies used were a mouse anti-GM130 (1:1000, BD Biosciences) and a rabbit anti-Dbnl (1:500, Proteintech). Before being stained with GM130, the sec- 

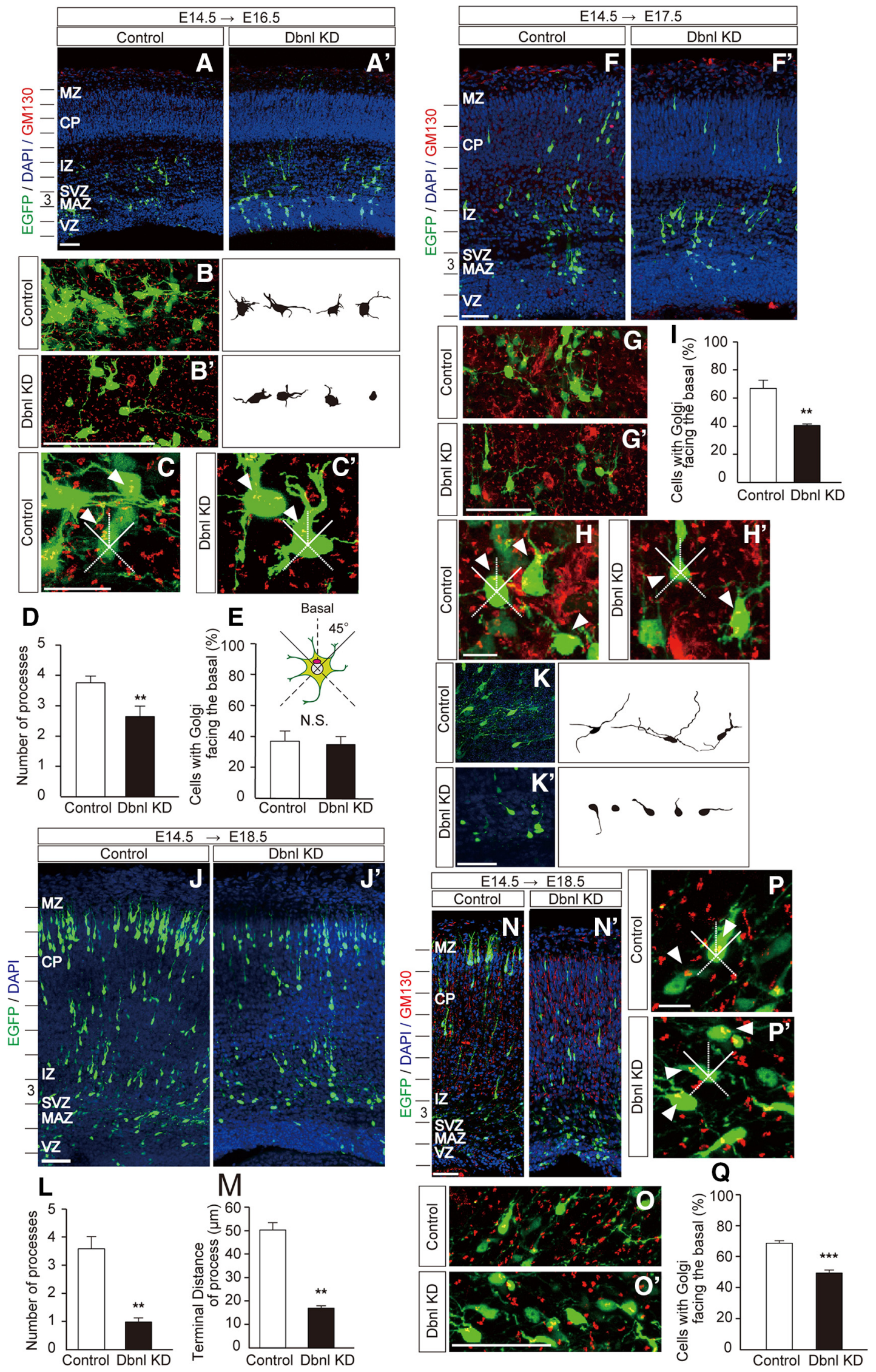

Figure 4. Dbnl is involved in the morphological alterations and polarization of neurons located in the MAZ/SVZ and IZ. $A, A^{\prime}$, Mouse embryonic brains at E14.5 were electroporated with the control $(\boldsymbol{A})$ or Dbnl-KD $\left(\boldsymbol{A}^{\prime}\right)$ vector together with pCAGGS-M-cre plus pCALNL-EGFP as visualized by the sparse labeling method, then fixed at E16.5 (Control, $N=4$ brains; Dbnl KD, $N=4$ brains). The entire cortex in the image was divided into 10 equally spaced bins (bin 1, deepest; bin 10, most superficial). $\boldsymbol{B}, \boldsymbol{B}^{\prime}$, Magnified images of the control (B) and Dbnl-KD neurons (Figure legend continues.) 
tions were incubated for $20 \mathrm{~min}$ in HistoVT One (Nakalai Tesque) at $70^{\circ} \mathrm{C}$.

Cell culture, transfection, and Western blot analysis. HEK293T cells were cultured in DMEM containing $100 \mathrm{U} / \mathrm{ml}$ penicillin and $100 \mu \mathrm{g} / \mathrm{ml}$ streptomycin supplemented with $10 \% \mathrm{FBS}$ in a humidified $5 \% \mathrm{CO}_{2}$ atmosphere at $37^{\circ} \mathrm{C}$. HEK293T cells were transfected with the plasmids using GeneJuice (Merck Millipore). After the cells were cultured for $48-72 \mathrm{~h}$, the cells were lysed with a cell lysis buffer (50 mm Tris- $\mathrm{HCl}, \mathrm{pH}$ $7.5,150 \mathrm{~mm} \mathrm{NaCl}, 1 \%$ NP-40, $0.3 \%$ Triton X-100, PhosSTOP Phosphatase Inhibitor Cocktail [Roche], and Complete Protease Inhibitor Cocktail [Roche]). The lysates were collected with a cell scraper, rotated for 30 min at $4^{\circ} \mathrm{C}$, sonicated, and solubilized with an SDS sample buffer $(100$ mм Tris- $\mathrm{HCl}, \mathrm{pH} 6.8,20 \%$ glycerol, $4 \%$ SDS, $0.005 \%$ bromophenol blue, and $200 \mathrm{~mm}$ dithiothreitol). After solubilized materials were boiled for 3 min at $96^{\circ} \mathrm{C}$, they were subjected to SDS-PAGE $(4 \%-15 \%$ acrylamide gel, Bio-Rad). Then, the gel was transferred on to a PVDF membrane using iBlot (Thermo Fisher Scientific). The blot was treated with a blocking buffer (PBS-Tw; 5\% BSA in PBS containing 0.05\% Tween 20) for $1 \mathrm{~h}$ at room temperature, then incubated overnight at $4^{\circ} \mathrm{C}$ with a primary antibody, followed by incubation with a secondary antibody for $1 \mathrm{~h}$. Finally, the blot was treated with ECL Plus Western blotting detection reagents (GE Healthcare). The signals were detected with a CCD camera (LAS4000mini; Fujifilm). The primary antibodies used were a rabbit antiDbnl (1:1000, Proteintech), a rabbit anti-GAPDH (1:1000, Cell Signaling Technology), a rabbit anti-FLAG (1:1000, Sigma-Aldrich), a mouse antiFLAG (1:1000, Sigma-Aldrich), and a mouse anti-phospho-tyrosine conjugated with HRP (PY-20; 1:1000, BD Biosciences). The secondary antibodies used were an anti-rabbit IgG conjugated with HRP, an antimouse IgG conjugated with HRP, or an anti-rat IgG conjugated with HRP (1:1000, Jackson ImmunoResearch Laboratories). In the case that the host of a primary antibody for Western blotting was the same as that of the antibody used for the immunoprecipitation assay, the TidyBlot Western blot detection reagent (1:200, Bio-Rad) was used instead of the secondary antibody to exclude the detection of IgG for immunoprecipitation.

\section{$\leftarrow$}

(Figure legend continued.) $\quad\left(\boldsymbol{B}^{\prime}\right)$ located in bin 3 of $\boldsymbol{A}$ and $\boldsymbol{A}^{\prime}$, and reconstructed shapes of the EGFP-positive neurons. $\boldsymbol{C}, \boldsymbol{C}^{\prime}$, Magnified images of the control $(\boldsymbol{C})$ and Dbnl-KD neurons $\left(\boldsymbol{C}^{\prime}\right)$ located in bin 3 of $\boldsymbol{A}$ and $\boldsymbol{A}^{\prime}$. Arrowheads indicate the Golgi apparatuses of the EGFP-positive neurons. Scale bars, $10 \mu \mathrm{m}$. $\boldsymbol{D}$, Quantitative comparison of the number of processes between the control neurons $(\boldsymbol{B})$ and Dbnl-KD neurons $\left(\boldsymbol{B}^{\prime}\right)(n=10-15$ neurons per brain were counted). $\boldsymbol{E}$, Quantitative comparison of the proportion of cells with their Golgi apparatus facing the basal part of the $C P$ between control neurons $(\boldsymbol{B})$ and Dbnl-KD neurons $\left(\boldsymbol{B}^{\prime}\right)(n=30-50$ neurons per brain were counted). $\boldsymbol{F}, \boldsymbol{F}^{\prime}$, Staining of the neuronal Golgi apparatuses (red) of the control neurons $(\boldsymbol{F})$ and Dbnl-KD neurons $\left(\boldsymbol{F}^{\prime}\right)$ transfected with pCAGGS-M-cre plus pCALNLEGFP atE14.5 and fixed at E17.5 (control, $N=3$ brains; Dbnl KD, $N=3$ brains). $\mathbf{G}, \mathbf{G}^{\prime}$, Magnified images of each of the control (G) and Dbnl-KD neurons $\left(\boldsymbol{G}^{\prime}\right)$ located in bin 3 of $\boldsymbol{F}$ and $\boldsymbol{F}^{\prime} . \boldsymbol{H}_{\boldsymbol{\prime}} \boldsymbol{H}^{\prime}$, Magnified images of the control $(\boldsymbol{H})$ and Dbnl-KD neurons $\left(\boldsymbol{H}^{\prime}\right)$ located in bin 3 of $\boldsymbol{F}$ and $\boldsymbol{F}^{\prime}$. Arrowheads indicate the location of Golgi apparatuses of the EGFP-positive neurons. Scale bars, $10 \mu \mathrm{m} . I$, Quantitative analysis of the control and Dbnl-KD neurons with the Golgi apparatuses facing the basal part of the CP ( $n=30-50$ neurons per brain were counted). $J, J^{\prime}$, Mouse embryonic brains were electroporated with PCAGGS-M-cre and pCALNL-EGFP with either the control $(J)$ or Dbnl-KD $\left(J^{\prime}\right)$ vector at E14.5 and fixed at E18.5 (control: $N=4$ brains; Dbnl KD: $N=3$ brains). The entire cortex in each image was divided into 10 equally spaced bins (bin 1 , deepest; bin 10, most superficial). $\boldsymbol{K}, \boldsymbol{K}^{\prime}$, Magnified images of the control ( $\boldsymbol{K}$ ) and Dbnl-KD neurons $\left(\boldsymbol{K}^{\prime}\right)$ located in bin 3 of $\boldsymbol{J}$ and $\boldsymbol{J}^{\prime}$, and reconstructed shapes of the EGFP-positive neurons in E18.5 cortices. $L, M$, Quantitative analyses of the number of processes elongating from the cell body $(\boldsymbol{L})$, and the length of the processes ( $n=10-15$ neurons per brain were counted) (M) between the control $(\boldsymbol{K})$ and Dbnl KD neurons $\left(\boldsymbol{K}^{\prime}\right)$. $\boldsymbol{N}, \boldsymbol{N}^{\prime}$, Staining of the Golgi apparatuses of the E14.5 mouse embryonic neurons electroporated with pCAGGS-M-cre plus pCALNL-EGFP together with the control $(\boldsymbol{N})$ or Dbnl-KD $\left(\boldsymbol{N}^{\prime}\right)$ plasmid (control: $N=3$ brains; Dbnl KD: $N=3$ brains). $\mathbf{0}, \mathbf{O}^{\prime}$, Magnified images of the control $(\boldsymbol{O})$ and Dbnl-KD neurons $\left(\boldsymbol{O}^{\prime}\right)$ located in bin 3 of $\boldsymbol{N}$ and $\boldsymbol{N}^{\prime}$, respectively. $\boldsymbol{P}, \boldsymbol{P}^{\prime}$, Magnified images of the control $(\boldsymbol{P})$ and Dbnl-KD neurons $\left(\boldsymbol{P}^{\prime}\right)$ located in bin 3 of $\boldsymbol{N}$ and $\boldsymbol{N}^{\prime}$. Arrowheads indicate the location of Golgi apparatuses of the EGFP-positive neurons. Scale bars, $10 \mu \mathrm{m}$. Q, Quantitative analysis of neurons with their Golgi apparatuses facing the basal part of the $C P$ in the control $(\boldsymbol{O})$ and Dbnl-KD neurons $\left(\boldsymbol{O}^{\prime}\right)(n=$ $30-50$ neurons per brain were counted). Error bar indicates mean \pm SEM. Student's $t$ test: ${ }^{* *} p<0.01 ;{ }^{* * *} p<0.001$. Scale bars, $50 \mu \mathrm{m}$.
Time-lapse imaging. The time-lapse imaging was performed as described previously, with slight modifications (Tabata and Nakajima, 2003; Kitazawa et al., 2014). Briefly, in utero electroporation was performed with pCAGGS-EGFP plus either the pSilencer-Dbnl shRNA or pSilencer-control into the ICR mouse cortex at E14.5. Then, $1.5 \mathrm{~d}$ later, coronal brain slices ( $250 \mu \mathrm{m}$ thickness) from the middle of three splits of the forebrain mounted in a low melting temperature agarose were cut with a vibratome (VT 1000S, Leica Microsystems). The slices were placed on a Millicell-CM membrane (pore size: $0.4 \mu \mathrm{m}$, Millipore) set on a glass base dish (AGC Techno Glass) and cultured in Neurobasal medium (Thermo Fisher Scientific) containing 2\% B27 (Thermo Fisher Scientific), 10\% FBS, and $500 \mu \mathrm{m}$ L-glutamine (Thermo Fisher Scientific). Then, the dishes were placed in a $40 \% \mathrm{O}_{2}$ incubator chamber (Tokai Hit) fitted onto a confocal microscope (SP8, Leica Microsystems). Then, $10-15$ optical $Z$-section images were acquired at $\sim 10 \mathrm{~min}$ intervals, and the focal planes were merged.

Immunoprecipitation assay. Cells were lysed with a cell lysis buffer (50 mм Tris-HCl, pH 7.5, 150 mм NaCl, 1\% NP-40, 0.3\% Triton X-100, PhosSTOP Phosphatase Inhibitor Cocktail [Roche Diagnostic], and Complete Protease Inhibitor Cocktail [Roche Diagnostic]), and collected with a cell scraper. After being rotated for $30 \mathrm{~min}$ at $4^{\circ} \mathrm{C}$, the lysates were sonicated and a protein assay was performed. Equal amount of protein was used for the immunoprecipitation assay using Dynabeads protein G (Thermo Fisher Scientific). The antibodies used were a rabbit anti-Dbnl (1:100, Proteintech) and a mouse anti-FLAG (1:200, Sigma-Aldrich). After the sample was washed 3 times with the cell lysis buffer, it was dissociated with an SDS sample buffer. Then, after the solubilized materials were boiled for $3 \mathrm{~min}$ at $96^{\circ} \mathrm{C}$, they were subjected to SDS-PAGE (4\%-15\% acrylamide gel, Bio-Rad).

Membrane $\mathrm{N}$-cadherin assay. E16 cerebral cortices were dissected and incubated in warm PBS $\left(37^{\circ} \mathrm{C}\right)$ for $15 \mathrm{~min}$ and dissociated mechanically by pipetting. Then, the cells were transfected with a Dbnl-KD vector using an electroporator (NEPA21; Nepagene). The dissociated cells were plated onto a 12 -well plate coated with poly-L-lysine $(0.1 \mathrm{mg} / \mathrm{ml}$, SigmaAldrich). Two days later, the cultured neurons were washed twice with warm DMEM, and N-cadherin antibody (guinea pig, $2 \mu \mathrm{g} / \mathrm{ml}$ in DMEM) was applied to the plate, followed by incubation for $15 \mathrm{~min}$ at $37^{\circ} \mathrm{C}$. After being washed 3 times with warm DMEM, the cells were lysed in an SDS sample buffer. Bound N-cadherin antibodies were detected using an anti-guinea-pig IgG H\&L (HRP) (1:1000, Jackson ImmunoResearch). Signals of membrane-localized N-cadherin were normalized to the signals of GAPDH.

Quantification and statistical analyses. For the microscopic examinations, images were obtained by using the FLUOVIEW FV1000 confocal microscope (Olympus). To analyze the distributions of the EGFP-labeled neurons in the cortex, the distance from the ventricular surface to the top of the $\mathrm{CP}$ (D1) and the distance from the ventricular surface to the EGFP-positive neurons (D2) were measured using the ImageJ software, and the relative bin position was calculated by dividing D2 by D1. Quantitative analyses of the cellular morphology and Western blots were performed using ImageJ software. All numerical data are indicated as mean \pm SEM. When comparing two groups, the Student's $t$ test was performed, provided that neither normality nor homogeneity of variances of the dataset was rejected by the Shapiro-Wilk normality test and Levene's test, respectively. In cases where normality of the data was rejected, the Mann-Whitney $U$ test was used as a nonparametric test. When comparing multiple groups, normality of the data was verified by the Shapiro-Wilk test, and homogeneity of variances among all groups was tested by Levene's test. When there was no rejection of either the normality or homogeneity of the variance of the datasets to be analyzed, ANOVA followed by the Tukey-Kramer test was performed. In all statistical tests above, the $\alpha$ value was set at 0.05 .

\section{Results}

Dbnl is expressed in the developing mouse cerebral cortex To investigate whether Dbnl is expressed in the developing mouse cerebral cortex, we performed immunohistochemistry using brain sections at E16.5 and P0.5. Dbnl was detected through- 
multipolar migration (Time-lapse imaging)

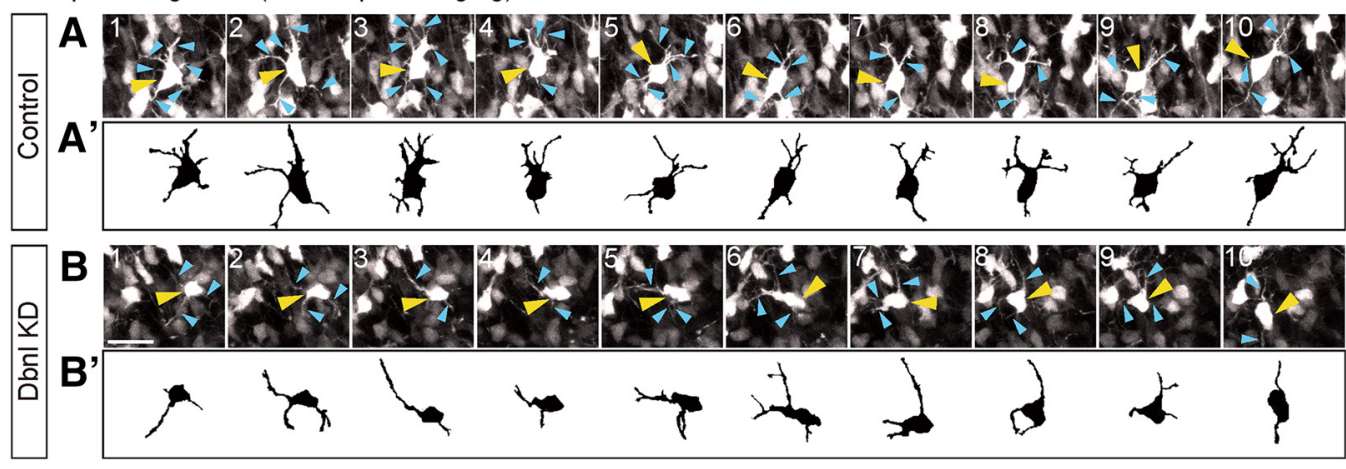

C

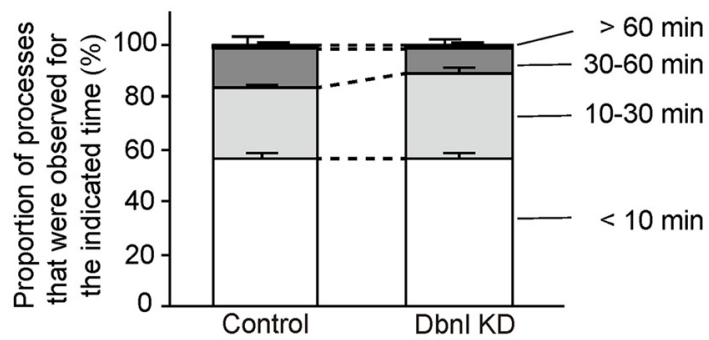

$\mathbf{F}$

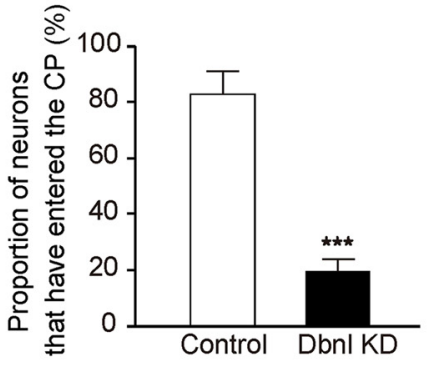

multipolar-bipolar transition (Time-lapse imaging)

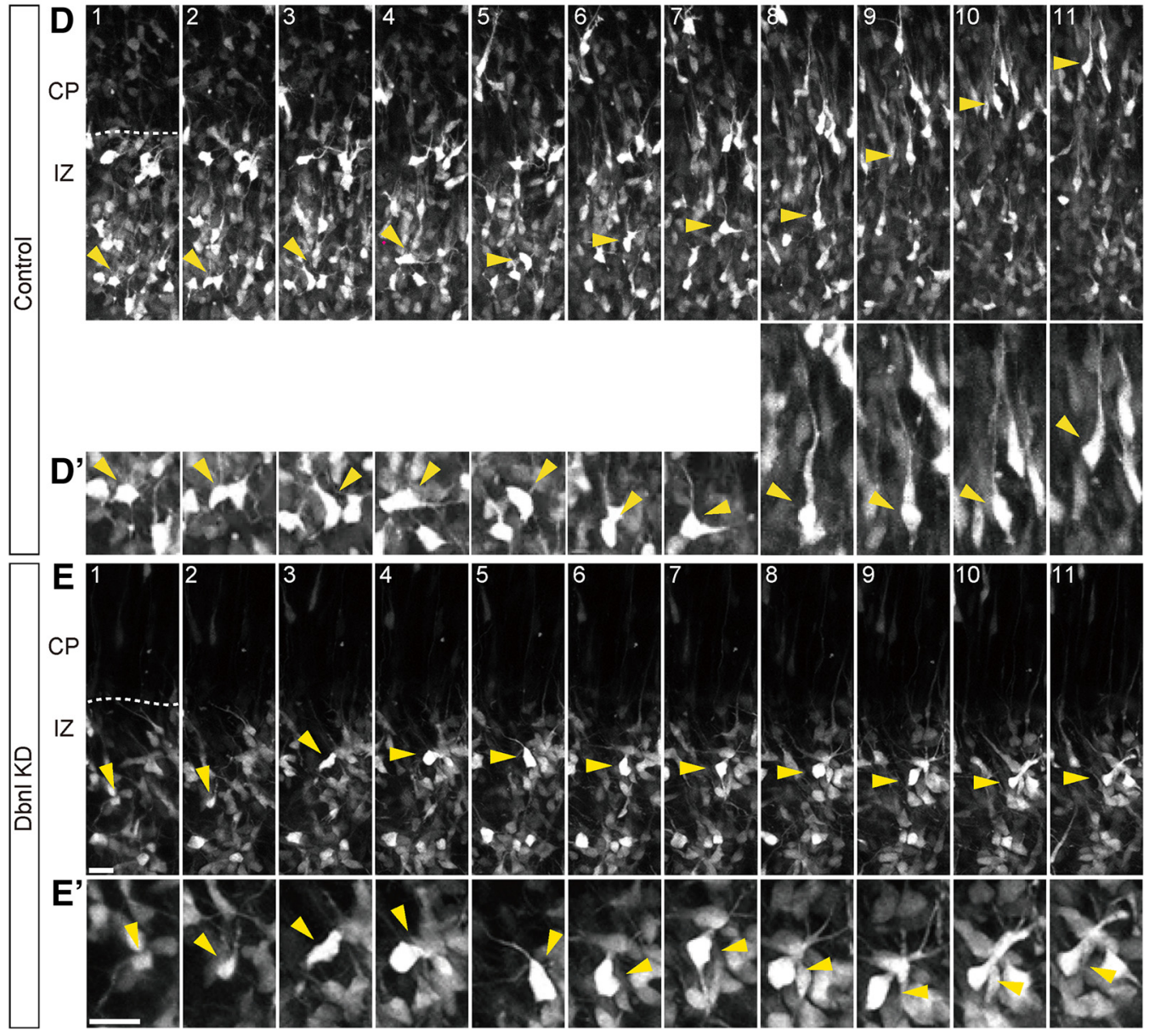

Figure 5. KD of Dbnl in migrating neurons restrains the entry of the neurons into the CP. $A, B$, Time-lapse images of migrating multipolar cells. The EGFP plasmid with either the $p$ Silencer-control $(\boldsymbol{A})$ or the Dbnl KD vector $(\boldsymbol{B})$ was electroporated into the cells along the ventricle at E14.5, and time-lapse images of the multipolar cells (yellow arrowheads) with multiple processes (cyan arrowheads) were obtained from the cortical slices of the electroporated brain after $2 \mathrm{~d}$. The time-lapse images were obtained every $10 \mathrm{~min}$ under a confocal microscope. The images obtained every $20 \mathrm{~min}$ are displayed. Reconstructed shapes of multipolar cells are shown in $\boldsymbol{A}^{\prime}$ (control) and $\boldsymbol{B}^{\prime}$ (Dbnl KD). $\boldsymbol{A}, \boldsymbol{B}$, Images are shown at the same magnification. Scale bar, $25 \mu \mathrm{m}$. $\boldsymbol{C}, \mathbf{Q u a n t i t a t i v e}$ analysis of the processes that were observed for the indicated time ( $N=4$ brains, $n=3-5$ neurons per brain, $n=8-20$ processes per neuron in each group). $\boldsymbol{D}, \boldsymbol{E}$, The EGFP plasmid with either the pSilencer-control $(\boldsymbol{D})$ or the Dbnl KD vector $(\boldsymbol{E})$ was electroporated at E14.5, and time-lapse images of the migrating cells (yellow arrowheads) were obtained (Figure legend continues.) 
out the neocortex, preferentially in the MZ and MAZ/SVZ at E16.5 (Fig. $1 A-A^{\prime \prime \prime}$ ). Dbnl was also detected at P0.5 (Fig. $1 B, B^{\prime}$ ). These results suggest that Dbnl plays important roles during cortical development.

\section{Dbnl-KD neurons show defects in neuronal migration during cortical development}

To examine whether Dbnl is required for neuronal migration in the mouse cortex, we constructed a Dbnl-KD vector, which significantly decreased the expression level of exogenous mouse Dbnl in HEK293T cells [Dbnl WT plus pSilencer-Dbnl (1): $0.046 \pm$ 0.012; Dbnl WT plus pSilencer3.0; (2): $1.00 \pm 0.00$; KD-resistant Dbnl plus pSilencer-Dbnl; (3): $1.20 \pm 0.25$; KDresistant Dbnl plus pSilencer3.0; (4): $0.91 \pm 0.16 ; N=3$ experiments; (1) vs $(2): p=4.20 \times 10^{-10}$; Figure $\left.2 A, B\right]$, as well as that of endogenous Dbnl in primary cultures of cortical neurons ( $\mathrm{pSi}$ lencer3.0: $1.00 \pm 0.00$; pSilencer-Dbnl: $0.45 \pm 0.12, N=3$ experiments, $p=0.023$; Fig. $2 C, D)$. This Dbnl-KD vector was introduced with an EGFP-expression vector into the VZ cells of the mouse cerebral cortex at E14.5 by in utero electroporation. At E17.5, both the control ( $N=10$ brains) and Dbnl-KD neurons ( $N=4$ brains) were found to be mainly located in the MAZ/SVZ and IZ, with no obvious differences in position between the two (Fig. 2E,F). On the other hand, at E18.5, by which time the majority of the control neurons ( $N=7$ brains) had entered the $\mathrm{CP}$ and reached beneath the MZ, many Dbnl-KD neurons $(N=$ 8 brains) were still found in the MAZ/SVZ and IZ (control vs Dbnl KD, bin 3: $p=0.001$; bin 10: $p=0.009$; Fig. 2G,H). Indeed, at P0.5, most Dbnl-KD neurons $(N=5$ brains $)$ still stayed in the SVZ and IZ (Fig. $2 L-N$ ). The Dbnl-KD phenotype was rescued by coexpression with a KD-resistant form of Dbnl at a low concentration $(0.5 \mu \mathrm{g} / \mu \mathrm{l})$ (control: $N=5$ brains; Dbnl KD $+\mathrm{KD}$ resistant Dbnl: $N=5$ brains) (bin3: control vs Dbnl KD, $p<$ 0.001; Dbnl KD + KD-resistant Dbnl vs Dbnl KD, $p=0.028$; bin 10: control vs Dbnl KD, $p<0.001$; Fig. $2 L-O)$. We confirmed that overexpression of the KD-resistant form of Dbnl at this concentration $(0.5 \mu \mathrm{g} / \mu \mathrm{l})$ had no effect on neuronal migration at this stage (data not shown).

This migration defect is not likely to represent a temporary delay of migration because many Dbnl-KD neurons were still located in the white matter (former IZ) at P2.5 (control: $N=7$ brains; Dbnl KD: $N=5$ brains; control vs Dbnl KD, bin $2: p=$ 0.003 ; bin 6: $p=0.048$; bin 8: $p=0.048$; bin 9: $p=0.01$; bin 10: $p=0.019$; Fig. $3 A, B$ ) and P5.5 (control: $N=6$ brains; Dbnl KD: $N=4$ brains; control vs Dbnl KD, bin 1: $p=0.004$; bin 2: $p=$ 0.004 ; bin $3: p=0.004$; bin $4: p=0.03$; bin $6: p=0.03$; bin $7: p=$ 0.03 ; bin 9: $p=0.004$; Fig. $3 C, D$ ). These results indicate that Dbnl is required for neuronal migration in the MAZ/SVZ and IZ.

\section{Dbnl regulates the morphology of multipolar neurons}

The accumulation of Dbnl-KD neurons in the IZ raised the possibility that Dbnl is required for multipolar-mode migration in the MAZ, or for transition from the multipolar to bipolar morphology beneath the CP. To observe the morphology of the Dbnl-KD neurons in the SVZ, neurons were transfected with a

\section{$\leftarrow$}

(Figure legend continued.) from the cortical slices of the electroporated brain after $2 \mathrm{~d}$. Magnified images of cells indicated by the arrowheads in $\boldsymbol{D}$ and $\boldsymbol{E}$ are shown in $\boldsymbol{D}^{\prime}$ and $\boldsymbol{E}^{\prime}$, respectively. The time-lapse images were taken every $10 \mathrm{~min}$. The images obtained every hour are displayed. $\boldsymbol{D}, \boldsymbol{E}$, Images are shown at the same magnification. Scale bar, $20 \mu \mathrm{m}$. $\boldsymbol{F}$, Quantitative analysis of the neurons that have entered the $C P(N=4$ brains, $n=17-25$ neurons per brain in each group). Error bar indicates mean \pm SEM. ${ }^{* *} p<0.001$ (Student's $t$ test).
Dbnl KD vector by in utero electroporation with sparse labeling, which was achieved by introducing a combination of a plasmid encoding EGFP downstream of a floxed stop cassette (pCALNLEGFP) $(2.0 \mu \mathrm{g} / \mu \mathrm{l})$ and a small amount of a Cre-expressing plasmid (pCAG-M-cre) $(2.0 \mathrm{ng} / \mu \mathrm{l})$ (Kitazawa et al., 2014). Two days after the electroporation, while the labeled neurons in control experiments ( $N=4$ brains) exhibited normal multipolar morphology with multiple thin processes in the MAZ (Fig. 4A,B), most of the Dbnl-KD neurons in this region showed poor formation of processes $\left(N=4\right.$ brains; Fig. $\left.4 A^{\prime}, B^{\prime}\right)$. Statistical analyses revealed a significantly reduced number of processes in the Dbnl-KD neurons compared with the control neurons (control: $3.75 \pm 0.22, N=4$ brains, $n=10-15$ neurons per brain were counted; Dbnl KD: $2.64 \pm 0.34, N=4$ brains, $n=10-15$ neurons per brain were counted; $p=0.0049$; Fig. $4 D$ ). Furthermore, we conducted time-lapse imaging of multipolar cells in the MAZ to compare the cellular dynamics between the control and Dbnl-KD neurons (control experiments, $N=4$ brains; Dbnl KD experiments, $N=4$ brains) (Fig. $5 A-B^{\prime}$; Movies 1,2). The control multipolar cells exhibited dynamic elongation and retraction of multiple processes as reported previously (Tabata and Nakajima, 2003; Noctor et al., 2004; Tabata et al., 2009). The Dbnl-KD cells showed frequent elongations and retractions of their processes (proportion of processes that were retracted within $10 \mathrm{~min}$ for control neurons: $56.48 \pm 1.79 \%, N=4$ brains, $n=3-5$ neurons per brain, $n=8-20$ processes per neuron; proportion of processes that were observed within $10 \mathrm{~min}$ for Dbnl-KD neurons: $56.26 \pm 2.75 \%, N=4$ brains, $n=3-5$ neurons per brain, $n=$ $8-20$ processes per neuron; $p=0.948$; Fig. $5 C$ ). These results suggest that Dbnl regulates the formation of processes in the multipolar neurons in the MAZ.

Next, to analyze the involvement of Dbnl in the polarization of neurons located around the MAZ, we compared the proportion of migrating neurons whose Golgi apparatus was positioned within 45 degrees from a line drawn perpendicular to the cortical surface between control and Dbnl-KD neurons in the MAZ/SVZ and IZ, by staining the cells with a Golgi apparatus marker, GM130 (Fig. $4 A-C^{\prime}, F-H^{\prime}, N-P^{\prime}$ ). Two days after in utero electroporation, there was no significant difference in the proportion of cells whose Golgi apparatuses were positioned on the CP side between the control (37.29 $\pm 6.49 \%, N=4$ brains, $n=30-50$ neurons per brain were counted) and Dbnl-KD neurons (35.00 $\pm 5.29 \%, N=4$ brains, $n=30-50$ neurons per brain were counted; control vs Dbnl KD, $p=0.65$; Fig. $4 E$ ). However, at $3 \mathrm{~d}$ after the electroporation, the proportion of neurons whose Golgi apparatuses were located on the CP side were significantly decreased in the Dbnl-KD neurons $(40.36 \pm 1.24 \% ; N=3$ brains, $n=30-50$ neurons per brain were counted) compared with the control neurons $(66.76 \pm 5.83 \%, N=3$ brains, $n=30-50$ neurons per brain were counted; control vs Dbnl KD, $p=0.0033$; Fig. $4 I)$. At $4 \mathrm{~d}$ after the electroporation, Dbnl-KD neurons left in the deep IZ exhibited fewer (control: $3.59 \pm 0.43, N=4$ brains, $n=$ 10-15 neurons per brain were counted; Dbnl KD: $0.98 \pm 0.15$, $N=3$ brains, $n=10-15$ neurons per brain were counted; control vs Dbnl KD, $p=0.0082$; Fig. $4 L$ ) and shorter processes (control: $50.20 \pm 3.15$; Dbnl KD: $16.90 \pm 1.06$; control vs Dbnl KD, $p=$ 0.0021 ; Fig. $4 M$ ) than the control neurons (Fig. $4 J-K^{\prime}$ ). In addition, the proportion of neurons whose Golgi apparatuses were facing the CP side was significantly decreased in the Dbnl-KD neurons ( $49.47 \pm 1.77 \%, N=3$ brains, $n=30-50$ neurons per brain were counted) compared with the control neurons (68.76 $\pm 1.55 \%, N=3$ brains, $n=30-50$ neurons per brain were counted; control vs Dbnl KD, $p=0.0082$; Fig. $4 N-Q)$. These 


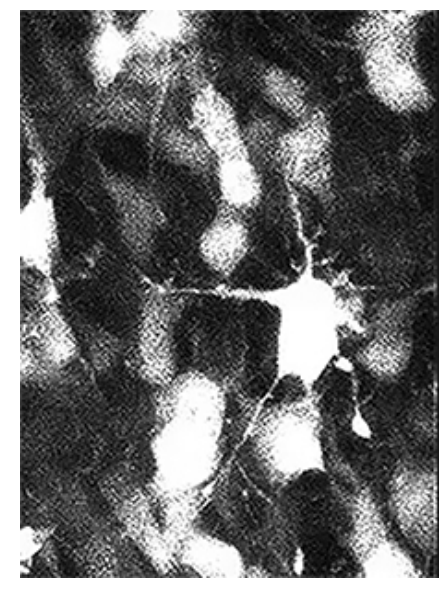

Movie 1. Time-lapse imaging of control multipolar cells labeled with EGFP. Images were captured every $10 \mathrm{~min}$. The movie corresponds to Figure $5 A$.

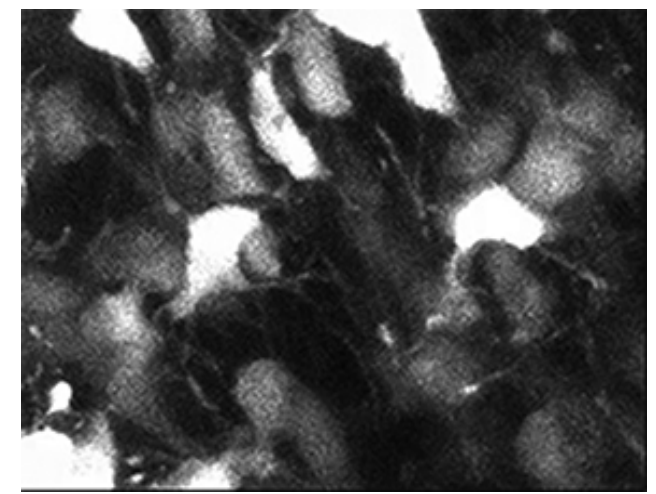

Movie 2. Time-lapse imaging of Dbnl-KD multipolar cells labeled with EGFP. Images were captured every $10 \mathrm{~min}$. The movie corresponds to Figure $5 B$.

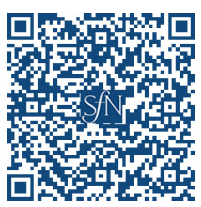

results indicate that Dbnl plays important roles in establishing neuronal morphology and polarization during neuronal migration in the developing cortex.

To investigate whether Dbnl is involved in the morphological transition of migrating neurons beneath the $\mathrm{CP}$, we performed time-lapse imaging of control or Dbnl-KD neurons visualized with EGFP. Control neurons with multipolar morphology gradually switched to a bipolar morphology, with a thick leading process, then entered the $\mathrm{CP}$ and climbed up in the $\mathrm{CP}$, one after the other, toward the brain surface (Fig. 5D-E'; Movies 3, 4). In contrast, while Dbnl-KD neurons also switched from a multipolar morphology to bipolar morphology, many of these neurons were unable to enter the $\mathrm{CP}$, resulting in pooling of these migrating neurons below the CP. When we counted the number of EGFP-labeled neurons in the IZ at the beginning of culture and calculated the proportion of neurons that have entered the $\mathrm{CP}$ by $36 \mathrm{~h}$ in vitro, a larger proportion of control neurons have entered the CP compared with the Dbnl-KD neurons (control: $82.66 \pm$ $8.13 \%, N=4$ brains, $n=17$ to 25 neurons per brain; Dbnl-KD:

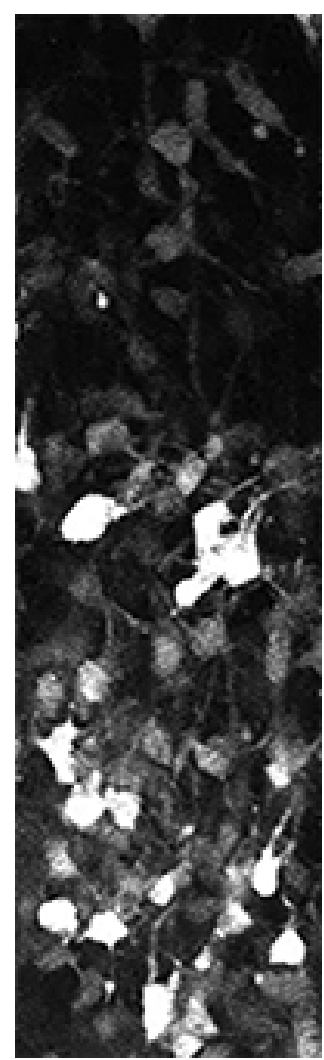

Movie 3. Time-lapse imaging of control migrating neurons labeled with EGFP. Images were captured every $10 \mathrm{~min}$. The movie corresponds to Figure $5 D$.

$19.63 \pm 4.17 \%, N=4$ brains, $n=20-25$ neurons per brain, $p=$ 0.00046 ; Fig. $5 F$ ). These results suggest that Dbnl is closely involved in regulating neuronal entry into the $\mathrm{CP}$.

\section{Phosphorylations of tyrosine residues 337 and 347 of Dbnl} are essential for migrating neurons to enter the $\mathrm{CP}$ In NIH3T3 fibroblasts, Dbnl is phosphorylated at Y337 and/or Y347 by a Src kinase (Boateng et al., 2012). In the developing cerebral cortex, Fyn kinase is thought to be dominant among the SFKs (Cooke and Perlmutter, 1989; Sasaki et al., 2002; Yuasa et al., 2004). Furthermore, Fyn-kinase-dead neurons exhibited defects in neuronal migration, especially during multipolarto-bipolar transition, abnormal neuronal morphology, and disorientation of their apical dendrites in the developing mouse cerebral cortex (Huang et al., 2017), which are, to some extent, similar to the observations for Dbnl-KD neurons in this study. Therefore, to identify whether Y337 and Y347 of Dbnl could also be essential for the phosphorylation by Fyn, we prepared vectors to express the phospho-resistant form of Dbnl at Y337 and/or Y347 (Dbnl Y337F, Dbnl Y347F or Dbnl 2F [Y337F and Y347F]) (Fig. 2I). These mutant forms of Dbnl were cotransfected into the HEK293T cells with the expression vectors of a constitutively active form of Fyn (Fyn-CA) or a kinase-dead form of Fyn. A lesser degree of phosphorylation by Fyn kinase of Dbnl Y337F and Dbnl Y347F was observed compared with that of the Dbnl WT. Moreover, double mutations at these tyrosine residues abolished the tyrosine phosphorylation of Dbnl by Fyn $[n=6$; Dbn] 


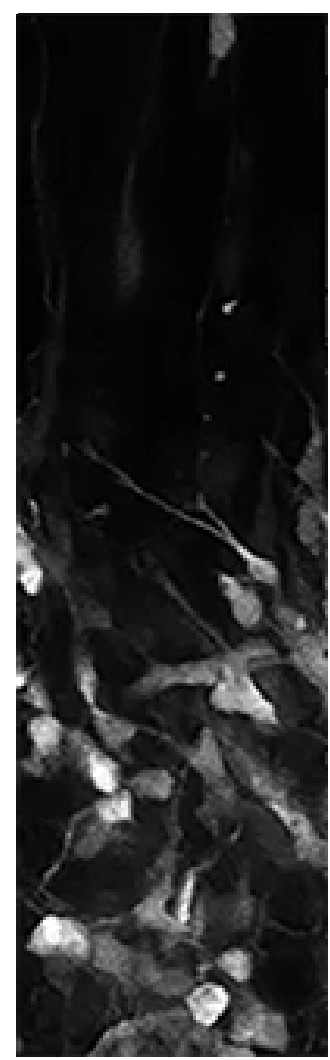

Movie 4. Time-lapse imaging of Dbnl-KD migrating neurons labeled with EGFP. Images were captured every $10 \mathrm{~min}$. The movie corresponds to Figure $5 E$.

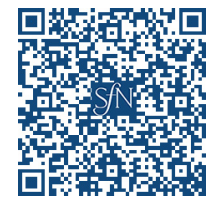

WT plus Fyn CA (1): $1.00 \pm 0.00$; Dbnl Y337F plus Fyn CA (2): $0.12 \pm 0.057$; Dbnl Y347F plus Fyn CA (3): $0.12 \pm 0.037$; Dbnl 2F plus Fyn CA (4): $0.030 \pm 0.0076$; (1) vs $(2): p=3.71 \times 10^{-7},(1)$ vs $(3): p=4.46 \times 10^{-8},(1)$ vs $(4): p=4.85 \times 10^{-9},(2)$ vs $(4)$ : $p=0.019$, (3) vs (4): $p=0.0013$; Figure $2 J, K)]$. These results indicate that Y337 and Y347 on Dbnl can be phosphorylated by Fyn in the HEK293T cells.

Next, to examine whether the phosphorylation of Y337 and Y347 on Dbnl is involved in neuronal migration, we introduced the Dbnl-KD vector with an expression vector for either Dbnl 2F or the phospho-mimic form of Dbnl (Dbnl 2E; Y337E and Y347E), in which the Y337 and Y347 residues were substituted with glutamic acid, into the cells in the VZ at E14.5 (control: $N=5$ brains; Dbnl KD: $N=5$ brains; Dbnl KD plus Dbnl 2F: $N=4$ brains; Dbnl KD plus Dbnl 2E: $N=7$ brains; Figure $2 L-O)$. Five days later, most control neurons reached beneath the MZ, whereas neurons with Dbnl 2F were still located beneath the $\mathrm{CP}$, which is similar to the migratory defect of the Dbnl KD neurons (bin3: control vs Dbnl 2F, $p<$ 0.001; Dbnl 2E vs Dbnl KD, $p=0.005$; Dbnl 2E vs Dbnl 2F, $p=$ 0.01 ; Fig. $2 N)$. On the other hand, most of the neurons with Dbnl 2E successfully reached beneath the MZ (bin 10: control vs Dbnl 2F, $p<0.001$; Fig. 2O). These results indicate that the phosphorylation of Y337 and Y347 of Dbnl is essential for the neurons to enter the CP.

\section{Dbnl controls neuronal migration} via $\alpha \mathrm{N}$-catenin/ $\mathrm{N}$-cadherin

It has been reported that trafficking of $\mathrm{N}$-cadherin to the neuronal plasma membrane is involved in the multipolar-tobipolar transition of migrating neurons (Jossin and Cooper, 2011). To examine whether Dbnl regulates the amount of $\mathrm{N}$-cadherin expressed on the plasma membrane, we performed an in vitro membrane $\mathrm{N}$-cadherin assay using an anti$\mathrm{N}$-cadherin antibody that recognizes the extracellular region of N-cadherin (Hara et al., 2016). Primary cultured cortical neurons were transfected with a Dbnl-KD vector, and the amount of $\mathrm{N}$-cadherin expressed on the plasma membrane was measured by quantifying the amount of $\mathrm{N}$-cadherin antibody bound to the cellular surface. The results revealed that the surface expression of $\mathrm{N}$-cadherin was significantly decreased by Dbnl KD (control: $1.00 \pm 0.00$; Dbnl KD: $0.30 \pm$ $0.17 ; N=4$ experiments; control vs Dbnl KD, $p=0.0057$; Fig. $6 A, B)$, indicating that Dbnl increases the amount of $\mathrm{N}$-cadherin expressed on the cellular surface.

Next, we investigated whether the migratory defect caused by Dbnl KD could be rescued by $\mathrm{N}$-cadherin overexpression. Moderate overexpression of $\mathrm{N}$-cadherin indeed rescued the migration defect caused by $\mathrm{Dbnl} \mathrm{KD}$, although the rescue effect was not complete (Fig. $6 C-F$ ). Dbnl is known to play a role in the reorganization of the actin cytoskeleton by interacting with actin filaments (Kessels et al., 2001). In addition, $\mathrm{N}$-cadherin also interacts with actin filaments through $\beta$-catenin and $\alpha$-catenin, and $\alpha \mathrm{N}$-catenin is a dominant member of the $\alpha$-catenin family expressed in the IZ of the developing cortex (Ajioka and Nakajima, 2005). Hence, we speculated whether Dbnl might also be involved in the $\alpha \mathrm{N}$-cateninmediated pathway. As expected, introduction of $\alpha \mathrm{N}$-catenin moderately rescued the migration defect caused by Dbnl KD. Furthermore, coexpression of both $\mathrm{N}$-cadherin and $\alpha \mathrm{N}$ catenin in the migrating neurons almost completely rescued the migration failure caused by Dbnl KD (bin3: control vs Dbnl KD, $p=0.015$; Dbnl KD vs Dbnl KD plus $\mathrm{N}$-cadherin and $\alpha \mathrm{N}$-catenin, $p=0.016$; bin 9: control vs Dbnl KD, $p=$ 0.007; Dbnl KD vs Dbnl KD plus $N$-cadherin, $p=0.020$; Dbnl KD plus $\mathrm{N}$-cadherin and $\alpha \mathrm{N}$-catenin: $p=0.026$; control: $N=$ 4 brains; Dbnl KD: $N=5$ brains; Dbnl KD plus N-cadherin: $N=4$ brains; Dbnl KD plus $\alpha \mathrm{N}$-catenin: $N=5$ brains; Dbnl KD plus $\mathrm{N}$-cadherin and $\alpha \mathrm{N}$-catenin: $N=8$ brains; Fig. $6 C-$ $F)$. These results suggest that Dbnl controls neuronal migration via regulating $\alpha \mathrm{N}$-catenin and $\mathrm{N}$-cadherin in the IZ of the developing mouse cerebral neocortex.

\section{Discussion}

Disruption of neuronal migration can cause various brain disorders, such as lissencephaly and subcortical band heterotopia. The precise molecular pathway based on the actin cytoskeleton that controls neuronal migration remains unknown until date, despite its importance. In this study, we found that Dbnl is essential for neuronal migration, neuronal process formation during multipolar migration, and polarization of migrating neurons in the MAZ/SVZ and IZ. The functioning of Dbnl in regulating neuronal migration appears to be dependent on the phosphorylation of Dbnl at tyrosine residues 337 and 347. Moreover, Dbnl controls neuronal migration through upregulating the cell surface expression of $\mathrm{N}$-cadherin in the developing cerebral cortex. 
A membrane $\mathrm{N}$-cadherin assay

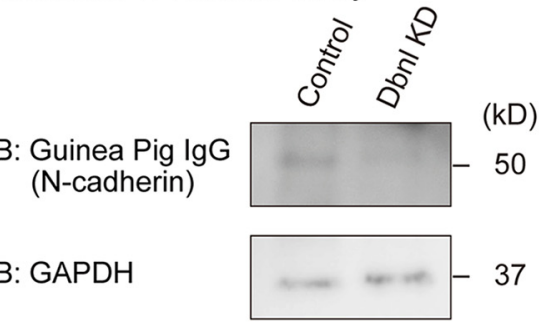

B

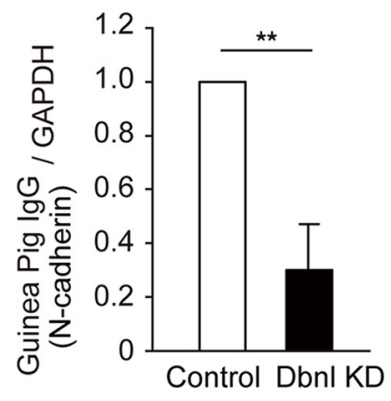

C
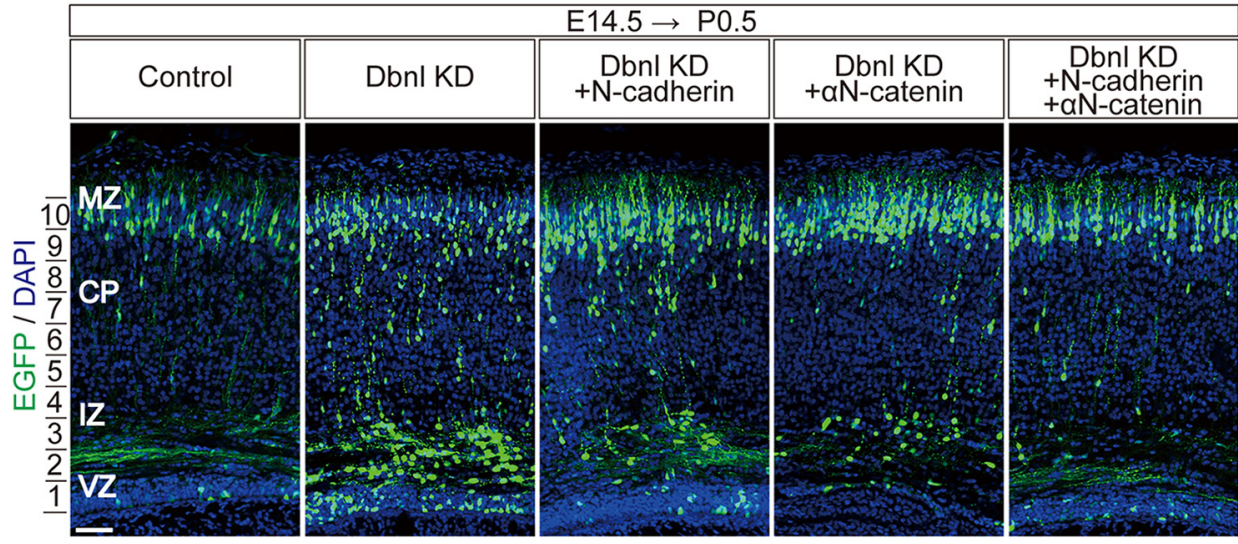

D

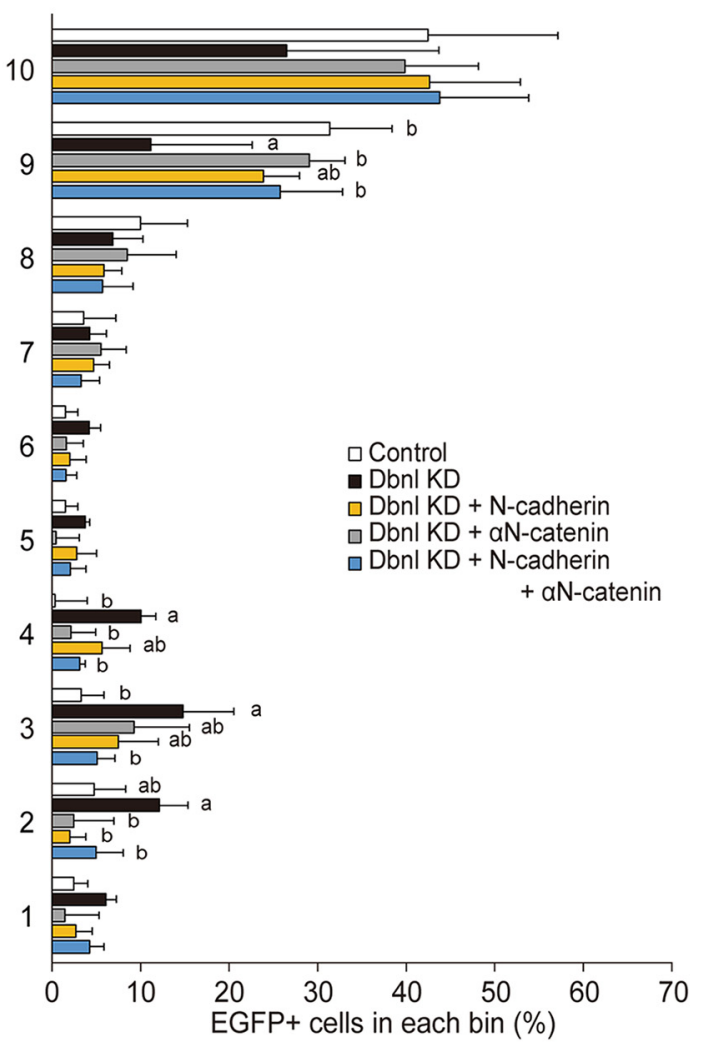

E

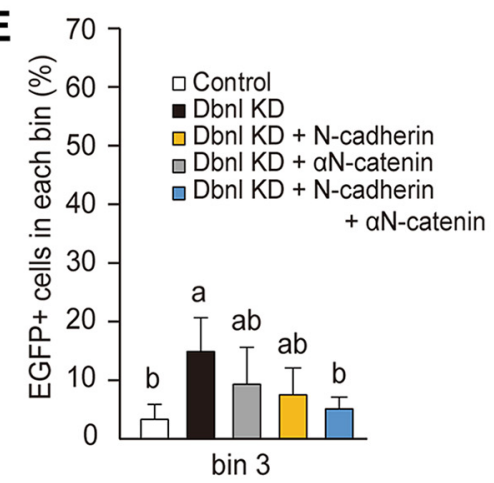

$\mathbf{F}$

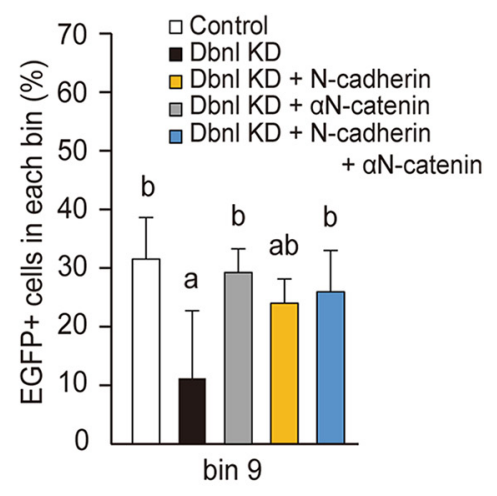

Figure 6. Dbnl regulates neuronal migration in the developing cortex through regulation of $\mathrm{N}$-cadherin and $\alpha \mathrm{N}$-catenin. $\boldsymbol{A}$, in vitro membrane $\mathrm{N}$-cadherin assay using primary cortical neurons at E16. The cultured neurons were treated with a guinea pig anti-N-cadherin antibody, and the amount of $\mathrm{N}$-cadherin expressed on the neuronal plasma membrane was measured by quantifying the amount of anti-N-cadherin antibody bound to the neurons. The cell lysates were subjected to Western blotting with an anti-guinea pig lgG antibody and an anti-GAPDH antibody as an internal control. B, A normalized graph represents the guinea pig $\lg G(\mathrm{~N}$-cadherin)/GAPDH ratio $(\boldsymbol{A})(\mathrm{N}=4$ experiments). $\mathrm{C}$, E14.5 mouse embryonic brains were electroporated with the Dbnl-KD vector and pCAGGS-EGFP plus pCAGGS-1 ( $N=5$ brains), pCAGGS-N-cadherin ( $N=4$ brains), pCAGGS- $\alpha N$-catenin $(N=5$ brains), or both of pCAGGS-N-cadherin and pCAGGS- $\alpha N$-catenin $(N=8$ brains) plasmids. pSilencer-control vector plus pCAGGS-1 and pCAGGS-EGFP were used as the controls ( $N=4$ brains). The brains were fixed at P0.5 and sectioned. Each section was stained with DAPI. $D$, Graph showing the proportion of EGFP-positive cells in each bin. White bar represents Control. Black bar represents Dbnl KD. Gray bar represents Dbnl KD + N-cadherin. Orange bar represents Dbnl $\mathrm{KD}+\alpha \mathrm{N}$-catenin. Blue bar represents Dbnl KD $+\mathrm{N}$-cadherin $+\alpha \mathrm{N}$-catenin. The entire cortex in each image was divided into 10 equally spaced bins (bin 1, (Figure legend continues.) 
Dbnl is known as an adaptor protein containing an ADF-H domain at its $\mathrm{N}$-terminal end, a proline-rich region, and an SH3 domain at its C-terminal end (Larbolette et al., 1999) (Fig. 2I). The $\mathrm{N}$ terminus of Dbnl is highly homologous with Drebrin, which is also a member of the ADF-H family and stabilizes F-actin in the brain (Shirao and Obata, 1985). Dbnl contains an SH3 domain and a proline-rich region, the Y337 and Y347 residues of which are phosphorylated by the SFKs in fibroblasts (Boateng et al., 2012), whereas Drebrin does not possess these domains. Our results suggest that the Y337 and 347 residues of Dbnl are involved in the Fyn-dependent phosphorylation of this protein (Fig. $2 I-K$ ); therefore, we assume that Dbnl could be a downstream molecule of Fyn, but not Drebrin.

Histological analyses of the Dbnl-KD neurons during neuronal migration and time-lapse imaging of these neurons suggest that Dbnl controls multiple events in migrating neurons located in the MAZ/SVZ and IZ of the developing cortex, as follows:

(1) process formation of multipolar neurons, (2) establishment of cell polarity, and (3) neuronal entry into the CP.

The process formation in multipolar neurons may be important for the investigation of environmental cues, for example, those associated with the direction of axonal growth (Tabata and Nakajima, 2003). One of the unique features of the processes of multipolar neurons is their dynamic movements, namely, frequent and repeated elongations and retractions (Tabata and Nakajima, 2003). Actin polymerization and depolymerization are likely to be involved in such dynamic movements. Dbnl is known to be an adaptor protein involved in the organization of the actin cytoskeleton. For example, Dbnl facilitates dendritogenesis and spine formation through interaction with F-actin and other molecules involved in organization of the actin cytoskeleton (Haeckel et al., 2008; Haag et al., 2012). Thus, Dbnl in the multipolar cells may regulate actin polymerization through interacting with $\mathrm{F}$-actin and related proteins, such as Arp2/3 complex and Cobl (Kessels et al., 2000).

Second, establishment of cell polarity is essential for morphological transformation of the migrating neurons. Our results showed that the proportion of neurons with their Golgi apparatus positioned on the $\mathrm{CP}$ side was significantly decreased in the Dbnl-KD neurons compared with the control neurons (Fig. 4G-I). The actin cytoskeleton is also important for regulation of the cellular polarity by the Rho-family GTPases, such as Cdc42 and Rac1 (Kodani et al., 2009; Tahirovic et al., 2010). Because Dbnl interacts with the Cdc42 GEF (Hou

\footnotetext{
$\leftarrow$

(Figure legend continued.) deepest; bin 10, most superficial). Statistically significant differences were observed in bin 2 (Dbnl KD vs Dbnl KD $+\mathrm{N}$-cadherin, ${ }^{* *} p=0.008$; Dbnl KD vs Dbnl $\mathrm{KD}+\alpha \mathrm{N}$-catenin, ${ }^{* *} p=0.003 ;$ Dbnl KD vs Dbnl KD $+\mathrm{N}$-cadherin $+\alpha \mathrm{N}$-catenin, ${ }^{*} p=$ 0.025 ), bin 3 (Control vs Dbnl KD, ${ }^{*} p=0.015$; Dbnl KD vs Dbnl KD $+\mathrm{N}$-cadherin $+\alpha \mathrm{N}$-catenin, ${ }^{*} p=0.016$ ), bin 4 (Control vs Dbnl KD, ${ }^{* *} p=0.001$; Dbnl KD vs Dbnl KD $+\alpha \mathrm{N}$-catenin, ${ }^{* *} p=$ 0.009 ; Dbnl KD vs Dbnl KD $+\mathrm{N}$-cadherin $+\alpha \mathrm{N}$-catenin, ${ }^{* *} p=0.007$ ), and bin 9 (Control vs Dbnl KD, ${ }^{* *} p=0.007$; Dbnl KD vs Dbnl KD $+\mathrm{N}$-cadherin, ${ }^{*} p=0.020$; Dbnl KD vs Dbnl KD + $\mathrm{N}$-cadherin $+\alpha \mathrm{N}$-catenin, $\left.{ }^{*} p=0.026\right) . \boldsymbol{E}, \boldsymbol{F}$, Graphs represent the proportion of EGFP-positive cells in bin $3(\boldsymbol{E})$ and bin $9(\boldsymbol{F})$. D, Different letters on the bars within the same bin indicate a statistically significant difference between the pair, whereas the same letter within the same bin or the absence of any letters within the bins indicates the absence of any statistically significant difference. Error bar indicates mean \pm SEM. Student's $t$ test or one-way ANOVA with Tukey-Kramer post hoc test: ${ }^{* *} p<0.01$. Scale bars, $50 \mu \mathrm{m}$.
}

et al., 2003), Dbnl may play a role in the regulation of neuronal polarity in cooperation with $\mathrm{Cdc} 42$. Moreover, we assume that Dbnl affects the formation of multipolar cell processes before the establishment of cell polarity because, when the number of multipolar cell processes were already decreased by $2 \mathrm{~d}$ after introduction of the Dbnl KD vector (Fig. $4 D$ ), we did not find any differences in the Golgi apparatus location compared with that in the control neurons (Fig. 4E).

The third important role of Dbnl in the migration of neurons is to promote entry of the neurons into the CP. Dbnl-KD neurons could undergo multipolar-to-bipolar transition in the IZ; however, the Dbnl-KD bipolar neurons could not locomote into the CP. Migrating neurons in the locomotion mode require $\mathrm{N}$-cadherin trafficking to be attached to the radial glial fibers (Kawauchi et al., 2010; Jossin and Cooper, 2011). Dbnl was originally identified by a phage-displayed screening for proteins containing the SH3 domain (Sparks et al., 1996). SH3-containing proteins, such as amphiphysin (Wigge et al., 1997), syndapin (Qualmann et al., 1999), Dynamin (Shupliakov et al., 1997), and Cortactin (Kaksonen et al., 2000), which are structurally similar to Dbnl, are known to be involved in endocytosis. Dbnl has also been shown to bind to Dynamin and play a role in endocytosis in cooperation with Dynamin (Kessels et al., 2001; He et al., 2015). Previously, we reported that the introduction of a dominant-negative form of Dynamin into migrating neurons also caused a migratory defect in the IZ (Kawauchi et al., 2010). Therefore, it is possible that $\mathrm{Dbnl}$ is involved in $\mathrm{N}$-cadherin recycling during neuronal migration. Indeed, we successfully demonstrated that Dbnl is required for upregulating the amount of N-cadherin expressed on the neuronal plasma membrane (Fig. 6A,B). It should be noted that the defect in multipolar-to-bipolar transition and entry into the CP caused by Dbnl KD was only partially rescued by overexpression of either $\mathrm{N}$-cadherin or $\alpha \mathrm{N}$-catenin, but completely rescued by coexpression of both $\mathrm{N}$-cadherin and $\alpha \mathrm{N}$-catenin (Fig. $6 C$ ). These results suggest that the interaction between $\mathrm{N}$-cadherin and $\alpha \mathrm{N}$-catenin under Dbnl signaling may be important for proper neuronal migration in the developing cortex.

In conclusion, we found that Dbnl controls multiple processes during neuronal migration in the developing cerebral cortex. Future studies will be required to understand the molecular machinery involved in these processes regulated by Dbnl.

\section{References}

Ajioka I, Nakajima K (2005) Switching of alpha-catenin from alphaEcatenin in the cortical ventricular zone to alphaN-catenin II in the intermediate zone. Brain Res Dev Brain Res 160:106-111. CrossRef Medline

Boateng LR, Cortesio CL, Huttenlocher A (2012) Src-mediated phosphorylation of mammalian Abp1 (DBNL) regulates podosome rosette formation in transformed fibroblasts. J Cell Sci 125:1329-1341. CrossRef Medline

Cooke MP, Perlmutter RM (1989) Expression of a novel form of the fyn proto-oncogene in hematopoietic cells. New Biol 1:66-74. Medline

Cotton PC, Brugge JS (1983) Neural tissues express high levels of the cellular src gene product pp60c-src. Mol Cell Biol 3:1157-1162. CrossRef Medline

Franco SJ, Martinez-Garay I, Gil-Sanz C, Harkins-Perry SR, Müller U (2011) Reelin regulates cadherin function via Dab1/Rap1 to control neuronal migration and lamination in the neocortex. Neuron 69:482-497. CrossRef Medline

Goroncy AK, Koshiba S, Tochio N, Tomizawa T, Sato M, Inoue M, Watanabe S, Hayashizaki Y, Tanaka A, Kigawa T, Yokoyama S (2009) NMR solution structures of actin depolymerizing factor homology domains. Protein Sci 18:2384-2392. CrossRef Medline 
Haag N, Schwintzer L, Ahuja R, Koch N, Grimm J, Heuer H, Qualmann B, Kessels MM (2012) The actin nucleator cobl is crucial for Purkinje cell development and works in close conjunction with the F-actin binding protein Abp1. J Neurosci 32:17842-17856. CrossRef Medline

Haeckel A, Ahuja R, Gundelfinger ED, Qualmann B, Kessels MM (2008) The actin-binding protein Abp1 controls dendritic spine morphology and is important for spine head and synapse formation. J Neurosci 28:1003110044. CrossRef Medline

Hara Y, Fukaya M, Hayashi K, Kawauchi T, Nakajima K, Sakagami H (2016) ADP ribosylation factor 6 regulates neuronal migration in the developing cerebral cortex through FIP3/arfophilin-1-dependent endosomal trafficking of N-cadherin. eNeuro 3:ENEURO.0148-16.2016. CrossRef Medline

He K, Xing R, Yan X, Tian A, Zhang M, Yuan J, Lv Z, Fang X, Li Z, Zhang Y (2015) Mammalian actin-binding protein 1/HIP-55 is essential for the scission of clathrin-coated pits by regulating dynamin-actin interaction. FASEB J 29:2495-2503. CrossRef Medline

Honda T, Nakajima K (2006) Mouse Disabled1 (DAB1) is a nucleocytoplasmic shuttling protein. J Biol Chem 281:38951-38965. CrossRef Medline

Hou P, Estrada L, Kinley AW, Parsons JT, Vojtek AB, Gorski JL (2003) Fgd1, the Cdc42 GEF responsible for faciogenital dysplasia, directly interacts with cortactin and mAbpl to modulate cell shape. Hum Mol Genet 12: 1981-1993. CrossRef Medline

Huang Y, Li G, An L, Fan Y, Cheng X, Li X, Yin Y, Cong R, Chen S, Zhao S (2017) Fyn regulates multipolar-bipolar transition and neurite morphogenesis of migrating neurons in the developing neocortex. Neuroscience 352:39-51. CrossRef Medline

Jossin Y, Cooper JA (2011) Reelin, Rap1 and N-cadherin orient the migration of multipolar neurons in the developing neocortex. Nat Neurosci 14:697-703. CrossRef Medline

Kaksonen M, Peng HB, Rauvala H (2000) Association of cortactin with dynamic actin in lamellipodia and on endosomal vesicles. J Cell Sci 113: 4421-4426. Medline

Kawauchi T, Sekine K, Shikanai M, Chihama K, Tomita K, Kubo K, Nakajima K, Nabeshima Y, Hoshino M (2010) Rab GTPases-dependent endocytic pathways regulate neuronal migration and maturation through $\mathrm{N}$-cadherin trafficking. Neuron 67:588-602. CrossRef Medline

Kessels MM, Engqvist-Goldstein AE, Drubin DG (2000) Association of mouse actin-binding protein 1 (mAbp1/SH3P7), an src kinase target, with dynamic regions of the cortical actin cytoskeleton in response to Rac1 activation. Mol Biol Cell 11:393-412. CrossRef Medline

Kessels MM, Engqvist-Goldstein AE, Drubin DG, Qualmann B (2001) Mammalian Abp1, a signal-responsive F-actin-binding protein, links the actin cytoskeleton to endocytosis via the GTPase dynamin. J Cell Biol 153:351-366. CrossRef Medline

Kitazawa A, Kubo K, Hayashi K, Matsunaga Y, Ishii K, Nakajima K (2014) Hippocampal pyramidal neurons switch from a multipolar migration mode to a novel "climbing" migration mode during development. J Neurosci 34:1115-1126. CrossRef Medline

Kodani A, Kristensen I, Huang L, Sütterlin C (2009) GM130-dependent control of Cdc42 activity at the Golgi regulates centrosome organization. Mol Biol Cell 20:1192-1200. CrossRef Medline

Lappalainen P, Kessels MM, Cope MJ, Drubin DG (1998) The ADF homology (ADF-H) domain: a highly exploited actin-binding module. Mol Biol Cell 9:1951-1959. CrossRef Medline

Larbolette O, Wollscheid B, Schweikert J, Nielsen PJ, Wienands J (1999) SH3P7 is a cytoskeleton adapter protein and is coupled to signal transduction from lymphocyte antigen receptors. Mol Cell Biol 19:1539-1546. CrossRef Medline

Martinez R, Mathey-Prevot B, Bernards A, Baltimore D (1987) Neuronal pp60c-src contains a six-amino acid insertion relative to its non-neuronal counterpart. Science 237:411-415. CrossRef Medline

Matsuda T, Cepko CL (2007) Controlled expression of transgenes introduced by in vivo electroporation. Proc Natl Acad Sci U S A 104:10271032. CrossRef Medline

Nadarajah B, Brunstrom JE, Grutzendler J, Wong RO, Pearlman AL (2001) Two modes of radial migration in early development of the cerebral cortex. Nat Neurosci 4:143-150. CrossRef Medline

Nakajima K, Mikoshiba K, Miyata T, Kudo C, Ogawa M (1997) Disruption of hippocampal development in vivo by CR-50 mAb against reelin. Proc Natl Acad Sci U S A 94:8196-8201. CrossRef Medline

Nam S, Kim D, Cheng JQ, Zhang S, Lee JH, Buettner R, Mirosevich J, Lee FY, Jove R (2005) Action of the src family kinase inhibitor, dasatinib (BMS-
354825), on human prostate cancer cells. Cancer Res 65:9185-9189. CrossRef Medline

Niwa H, Yamamura K, Miyazaki J (1991) Efficient selection for highexpression transfectants with a novel eukaryotic vector. Gene 108:193199. CrossRef Medline

Noctor SC, Martínez-Cerdeno V, Ivic L, Kriegstein AR (2004) Cortical neurons arise in symmetric and asymmetric division zones and migrate through specific phases. Nat Neurosci 7:136-144. CrossRef Medline

Nuriya M, Huganir RL (2006) Regulation of AMPA receptor trafficking by N-cadherin. J Neurochem 97:652-661. CrossRef Medline

Qualmann B, Roos J, DiGregorio PJ, Kelly RB (1999) Syndapin I, a synaptic dynamin-binding protein that associates with the neural Wiskott-Aldrich Syndrome protein. Mol Biol Cell 10:501-513. CrossRef Medline

Rakic P (1972) Mode of cell migration to the superficial layers of fetal monkey neocortex. J Comp Neurol 145:61-83. CrossRef Medline

Sasaki Y, Cheng C, Uchida Y, Nakajima O, Ohshima T, Yagi T, Taniguchi M, Nakayama T, Kishida R, Kudo Y, Ohno S, Nakamura F, Goshima Y (2002) Fyn and Cdk5 mediate semaphorin-3A signaling, which is involved in regulation of dendrite orientation in cerebral cortex. Neuron 35:907-920. CrossRef Medline

Sekine K, Honda T, Kawauchi T, Kubo K, Nakajima K (2011) The outermost region of the developing cortical plate is crucial for both the switch of the radial migration mode and the Dab1-dependent "inside-out" lamination in the neocortex. J Neurosci 31:9426-9439. CrossRef Medline

Sekine K, Kawauchi T, Kubo K, Honda T, Herz J, Hattori M, Kinashi T, Nakajima K (2012) Reelin controls neuronal positioning by promoting cell-matrix adhesion via inside-out activation of integrin alpha5beta1. Neuron 76:353-369. CrossRef Medline

Shirao T, Obata K (1985) Two acidic proteins associated with brain development in chick embryo. J Neurochem 44:1210-1216. CrossRef Medline

Shupliakov O, Löw P, Grabs D, Gad H, Chen H, David C, Takei K, De Camilli P, Brodin L (1997) Synaptic vesicle endocytosis impaired by disruption of dynamin-SH3 domain interactions. Science 276:259-263. CrossRef Medline

Soriano P, Montgomery C, Geske R, Bradley A (1991) Targeted disruption of the c-src proto-oncogene leads to osteopetrosis in mice. Cell 64:693702. CrossRef Medline

Sparks AB, Hoffman NG, McConnell SJ, Fowlkes DM, Kay BK (1996) Cloning of ligand targets: systematic isolation of $\mathrm{SH} 3$ domain-containing proteins. Nat Biotechnol 14:741-744. CrossRef Medline

Stein PL, Vogel H, Soriano P (1994) Combined deficiencies of src, Fyn, and Yes tyrosine kinases in mutant mice. Genes Dev 8:1999-2007. CrossRef Medline

Sudol M, Alvarez-Buylla A, Hanafusa H (1988) Differential developmental expression of cellular yes and cellular src proteins in cerebellum. Oncogene Res 2:345-355. Medline

Tabata H, Nakajima K (2001) Efficient in utero gene transfer system to the developing mouse brain using electroporation: visualization of neuronal migration in the developing cortex. Neuroscience 103:865-872. CrossRef Medline

Tabata H, Nakajima K (2003) Multipolar migration: the third mode of radial neuronal migration in the developing cerebral cortex. J Neurosci 23:9996-10001. CrossRef Medline

Tabata H, Nakajima K (2008) Labeling embryonic mouse central nervous system cells by in utero electroporation. Dev Growth Differ 50:507-511. CrossRef Medline

Tabata H, Kanatani S, Nakajima K (2009) Differences of migratory behavior between direct progeny of apical progenitors and basal progenitors in the developing cerebral cortex. Cereb Cortex 19:2092-2105. CrossRef Medline

Tahirovic S, Hellal F, Neukirchen D, Hindges R, Garvalov BK, Flynn KC, Stradal TE, Chrostek-Grashoff A, Brakebusch C, Bradke F (2010) Rac1 regulates neuronal polarization through the WAVE complex. J Neurosci 30:6930-6943. CrossRef Medline

Vartiainen MK, Mustonen T, Mattila PK, Ojala PJ, Thesleff I, Partanen J, Lappalainen P (2002) The three mouse actin-depolymerizing factor/cofilins evolved to fulfill cell-type-specific requirements for actin dynamics. Mol Biol Cell 13:183-194. CrossRef Medline

Wigge P, Vallis Y, McMahon HT (1997) Inhibition of receptor-mediated endocytosis by the amphiphysin SH3 domain. Curr Biol 7:554-560. CrossRef Medline

Yuasa S, Hattori K, Yagi T (2004) Defective neocortical development in fyn-tyrosine-kinase-deficient mice. Neuroreport 15:819-822. CrossRef Medline 